\title{
Astragaloside IV Attenuates Myocardial Ischemia-Reperfusion Injury from Oxidative Stress by Regulating Succinate, Lysophospholipid Metabolism, and ROS Scavenging System
}

\author{
Miaomiao Jiang $\mathbb{D}^{1,2}$ Jingyu Ni, ${ }^{1,2}$ Yuanlin Cao, ${ }^{1,2}$ Xiaoxue Xing, ${ }^{1,2}$ Qian $\mathrm{Wu}^{3}$ \\ and Guanwei Fan ${ }^{1,2,4}$ \\ ${ }^{1}$ Tianjin State Key Laboratory of Modern Chinese Medicine, Tianjin University of Traditional Chinese Medicine, Tianjin, China \\ ${ }^{2}$ Tianjin Key Laboratory of Translational Research of TCM Prescription and Syndrome, Tianjin, China \\ ${ }^{3}$ Shanghai Center for Bioinformation Technology, Shanghai 201203, China \\ ${ }^{4}$ First Teaching Hospital of Tianjin University of Traditional Chinese Medicine, Tianjin Key Laboratory of Translational Research of \\ TCM Prescription and Syndrome, Tianjin, China
}

Correspondence should be addressed to Guanwei Fan; fgw1005@163.com

Received 26 November 2018; Revised 24 February 2019; Accepted 24 March 2019; Published 24 June 2019

Academic Editor: Silvana Hrelia

Copyright @ 2019 Miaomiao Jiang et al. This is an open access article distributed under the Creative Commons Attribution License, which permits unrestricted use, distribution, and reproduction in any medium, provided the original work is properly cited.

Astragaloside IV is one of the main active ingredients isolated from Astragalus membranaceus. Here we confirmed its protective effect against cardiac ischemia-reperfusion (I/R) injury and aimed to investigate the potential molecular mechanisms involved. Pretreatment of ex vivo and in vivo I/R-induced rat models by astragaloside IV significantly prevented the ratio of myocardium infarct size, systolic and diastolic dysfunction, and the production of creatine kinase and lactate dehydrogenase. Metabolic analyses showed that I/R injury caused a notable reduction of succinate and elevation of lysophospholipids, indicating excessive reactive oxygen species (ROS) generation driven by succinate's rapid reoxidization and glycerophospholipid degradation. Molecular validation mechanistically revealed that astragaloside IV stimulated nuclear factor (erythroid-derived 2)-like 2 (Nrf2) released from Kelch-like ECH-associated protein 1 (Keap1) and translocated to the nucleus to combine with musculoaponeurotic fibrosarcoma (Maf) to initiate the transcription of antioxidative gene heme oxygenase-1 (HO-1), which performed a wide range of ROS scavenging processes against pathological oxidative stress in the hearts. As expected, increasing succinate and decreasing lysophospholipid levels were observed in the astragaloside IV-pretreated group compared with the I/R model group. These results suggested that astragaloside IV ameliorated myocardial I/R injury by modulating succinate and lysophospholipid metabolism and scavenging ROS via the Nrf2 signal pathway.

\section{Introduction}

In recent years, ischemic cardiomyopathy has been a public health concern with a rising incidence that results in high morbidity and mortality worldwide [1]. Acute myocardial infarction (AMI) has the most severe effect, and the aftereffect of cardiac disease happens in the wake of interruption on the blood supply to the myocardium. Timely reperfusion for ST-elevation myocardial infarction is the cornerstone of treatment to reduce the mortality for patients with AMI in the last decades [2]. Inevitably, reoxygenation of hypoxic tissue exacerbates injury experienced during the ischemic epi- sode, which is further referred to as myocardial ischemiareperfusion (I/R) injury $[3,4]$. In clinical practice, I/R injury influences the therapeutic effect of the reperfusion strategy delivered to patients and has seriously adverse consequences, so that adjunctive medications aimed at reducing I/R injury are urgently needed.

Oxidative stress is one of the main pathogenic mechanisms of myocardial I/R injury [5]. Under normal conditions, a small amount of free radicals produced by oxidative metabolism in the organism can be removed in time by its own antioxidant system. During reperfusion of the ischemic myocardium, oxygen free radicals occur explosively and then 
initiate oxidative damage through their accumulation in the heart, which further results in destruction of oxidation balance and antioxidant systems, cell death, and aberrant immune responses [6,7]. Study evidence has demonstrated that metabolism provides substrates for reactive oxygen species (ROS), and thus, ROS generation is significantly influenced by these metabolic substrates, such as glucose, lipids, succinate, acetoacetate, choline, and dimethylglycine [811]. Metabolic dysfunction in tissues and organs caused by I/R injury has been manifested via the changes of metabolic substances in the body fluid or tissue [12], indicating that modulating metabolic substrate utilization in the myocardium would be an interesting therapeutic strategy for attenuation of ROS-related biochemical and functional damage.

Astragaloside IV is one of the main active ingredients in Astragalus membranaceus with various activities such as antitumor [13], anti-inflammation [14], antioxidation [15], antidiabetes [16], anticerebral ischemia [17], and anticardiovascular diseases [18]. Potential mechanisms of its protective effect on the heart mainly involve regulating the expression of ATP-sensitive potassium channel subunits [19] and levels of ATP, ADP, and AMP in the myocardium [20], stimulating angiogenesis and accumulation of nitric oxide (NO) through Janus kinase 2 (JAK2)/signal transducer and activator of transcription 3 (STAT3) and the extracellular signalregulated kinase 1/2 (ERK1/2) signaling pathway [21], upregulating the hypoxia-inducible factor- $1 \alpha$ (HIF- $1 \alpha$ ) expression [22] and preventing mitochondrial permeability transition pores from opening up by inactivating the glycogen synthase enzyme-3 $\beta$ (GSK-3 $\beta)$ [23]. Whether astragaloside IV mediate the metabolic response to myocardial I/R injury and the specific molecular mechanisms responsible for scavenging effect to excessive ROS are not completely understood.

In this study, we have demonstrated that astragaloside IV alleviates cardiac damage induced by $\mathrm{I} / \mathrm{R}$ using ex vivo and in vivo rat models. We further systematically investigate the effects of astragaloside IV intake on the metabolism of the rat myocardium using ${ }^{1} \mathrm{H}$ nuclear magnetic resonance $\left({ }^{1} \mathrm{H}\right.$ NMR) spectroscopy in conjunction with ultra-highperformance liquid chromatography with quadrupoleorbitrap mass spectrometry (UHPLC-MS). A global metabolic profiling approach is used to screen for potential metabolites that may be associated with I/R-induced oxidative stress, and the alternations of these metabolites are employed to evaluate the cardioprotective effect of astragaloside IV. Furthermore, we also assess whether astragaloside IV intervention can eliminate excessive ROS by regulating oxidative stress-related proteins. Taken all together, this study provides extensive information of astragaloside IV against myocardial $\mathrm{I} / \mathrm{R}$ injury that reveals the details of metabolism pathways and the enzymology involved.

\section{Materials and Methods}

2.1. Animals. Male Sprague-Dawley (SD) rats (SPF level, weighting $320 \pm 20 \mathrm{~g}$ ) were purchased from Beijing HFK Bioscience Co. Ltd., China (license No. SCXk2014-0004). All procedures were approved by the Animal Care and Use Committee of Tianjin University of Traditional Chinese
Medicine and were in accordance with the Guide for the Care and Use of Laboratory Animals. The rats were housed in cages at a temperature of $22 \pm 2^{\circ} \mathrm{C}$ and humidity of $40 \pm 5 \%$ with standard diet and water available ad libitum on a 12hour light/dark cycle. The animals were fasted for 12 hours before the experiment but allowed free access to water.

2.2. Chemicals. Astragaloside IV (purity $\geq 99 \%$ ) was obtained from Shanghai Winherb Medical Technology Co. Ltd. (Shanghai, China). Deuterium oxide $\left(\mathrm{D}_{2} \mathrm{O}, 99.9 \%\right.$ atom $\% \mathrm{D})$ and 3-(trimethylsilyl)-propionic-2,2,3,3- $d_{4}$ acid sodium salt (TSP- $d_{4}, 98 \%$ atom \%D) were purchased from SigmaAldrich (St. Louis, MO, USA). Lysophosphatidylcholine (12:0) and phosphatidylcholine (11:0/11:0) were purchased from Avanti Polar Lipids (Birmingham, AL, USA).

2.3. Langendorff Heart Experiments. A total of 60 rats were anesthetized with $5 \%$ chloral hydrate ( $300 \mathrm{mg} / \mathrm{kg}$ i.p.). Thoracotomy was performed, and hearts were rapidly excised into an ice-cold Krebs-Henseleit (KH) buffer $(118 \mathrm{mM} \mathrm{NaCl}$, $24.0 \mathrm{mM} \mathrm{NaHCO}{ }_{3}, 4.7 \mathrm{mM} \mathrm{KCl}, 1.2 \mathrm{mM} \mathrm{MgSO}_{4}, 1.2 \mathrm{mM}$ $\mathrm{KH}_{2} \mathrm{PO}_{4}, 11.1 \mathrm{mM}$ glucose, and $2.5 \mathrm{mM} \mathrm{CaCl}$ ) at $\mathrm{pH}$ 7.4. After removal of the lungs and surrounding tissues, the aorta was attached to a perfusion apparatus where hearts were perfused at a constant pressure of $65 \mathrm{mmHg}$ with $37^{\circ} \mathrm{C} \mathrm{KH}$ buffer in carbogen $\left(95 \% \mathrm{O}_{2}\right.$ and $\left.5 \% \mathrm{CO}_{2}\right)$. After $20 \mathrm{~min}$ recovery of function and stabilization of rhythm, the hearts were separated into four groups: 60 min normoxic perfusion (control group), 10 min normoxic perfusion followed by 20 min global ischemia plus $30 \mathrm{~min}$ reperfusion (I/R group), and 10 min perfusion with $0.05 \mu \mathrm{M}$ astragaloside IV in $\mathrm{KH}$ buffer followed by $20 \mathrm{~min}$ global ischemia plus $30 \mathrm{~min}$ reperfusion (AstraIV group). The experimental design is shown in Figure 1(a). At the end of the experiment, hearts were snapfrozen in liquid nitrogen and sorted at $-80^{\circ} \mathrm{C}$.

The infracted areas were displayed as the area unstained by 2,3,5-triphenyltetrazolium chloride (TTC) and quantified by ImageJ software. Cardiac function parameters were monitored before ischemia and each time period of reperfusion $(5,10,20$, and $30 \mathrm{~min})$, including heart rate (HR), coronary flow, left ventricular development pressure (LVDP), left ventricular end diastolic pressure, rate pressure product (RPP), left ventricular maximum upstroke velocity $\left(+\mathrm{d} p / \mathrm{d} t_{\max }\right)$, and left ventricular maximum descent velocity $\left(-\mathrm{d} p / \mathrm{d} t_{\max }\right)$. These parameters were recorded continuously on a computer using a PowerLab data acquisition system (8SP Chart 7 software; ADInstruments, Castle Hill, Australia). Levels of creatine kinase $(\mathrm{CK})$, lactate dehydrogenase $(\mathrm{LDH})$, superoxide dismutase (SOD), malondialdehyde (MDA), and succinate dehydrogenase $(\mathrm{SDH})$ were determined by commercial kits according to the manufacturer's instructions on automatic biochemical detector (Microlab 300, Holland Rittal Science Co. Ltd., Holland) and microplate reader (EnSpire, PerkinElmer Co. Ltd., USA).

2.4. Metabolic Profiling Based on NMR Measurements. Frozen heart samples were ground into powder on dry ice, 50 $\mathrm{mg}$ of which was extracted with cold methanol and water $(2: 1, v / v)$ for 3 times. Combined extracts were dried in 


\begin{tabular}{|c|c|c|c|c|}
\hline Control & Baseline & $\mathrm{KH}$ & $\mathrm{KH}$ & $\mathrm{KH}$ \\
\hline $\mathrm{I} / \mathrm{R}$ & Baseline & $\mathrm{KH}$ & Ischemia & Reperfusion \\
\hline \multirow[t]{2}{*}{ AstraIV } & Baseline & Drug & Ischemia & Reperfusion \\
\hline & $20 \mathrm{~min}$ & $\overrightarrow{10 \mathrm{~min}}$ & $20 \mathrm{~min}$ & $30 \mathrm{~min}$ \\
\hline
\end{tabular}

(a)

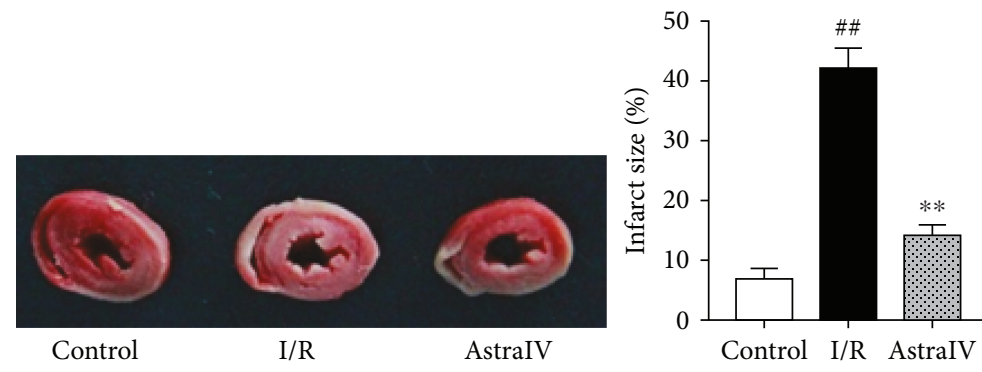

(b)

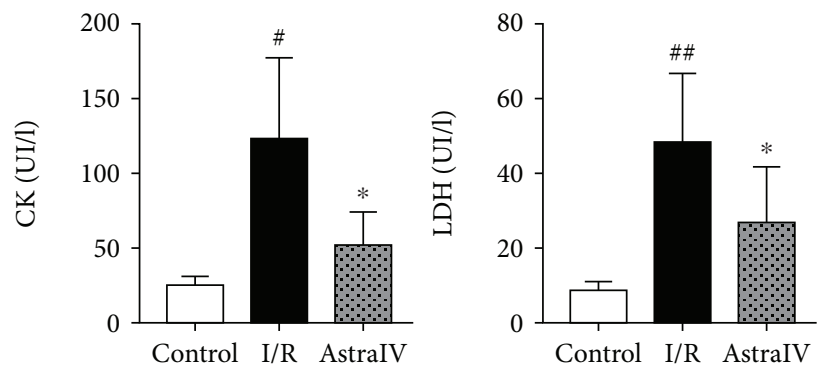

(c)
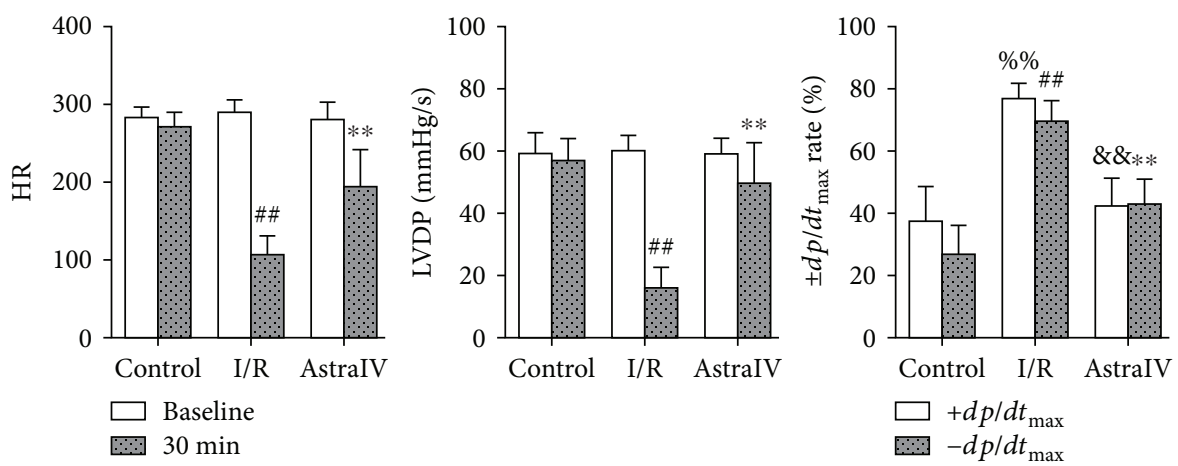

(d)
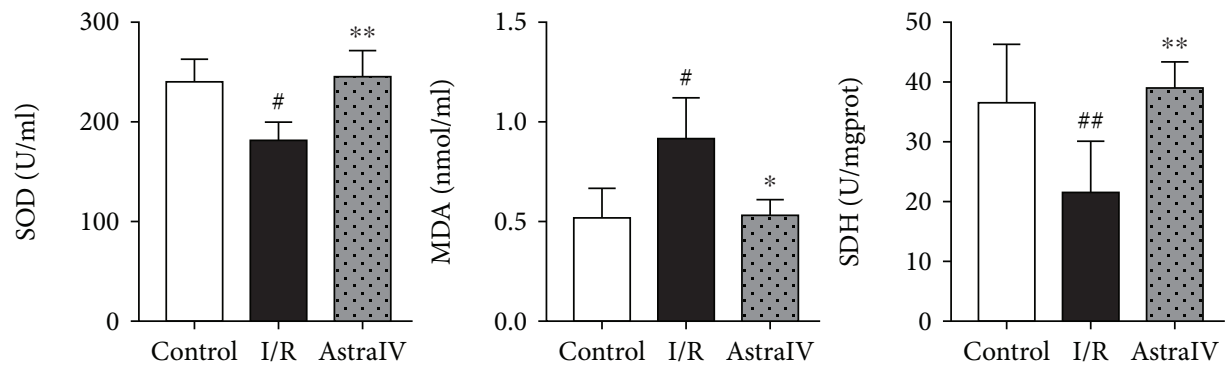

(e)

Figure 1: Continued. 

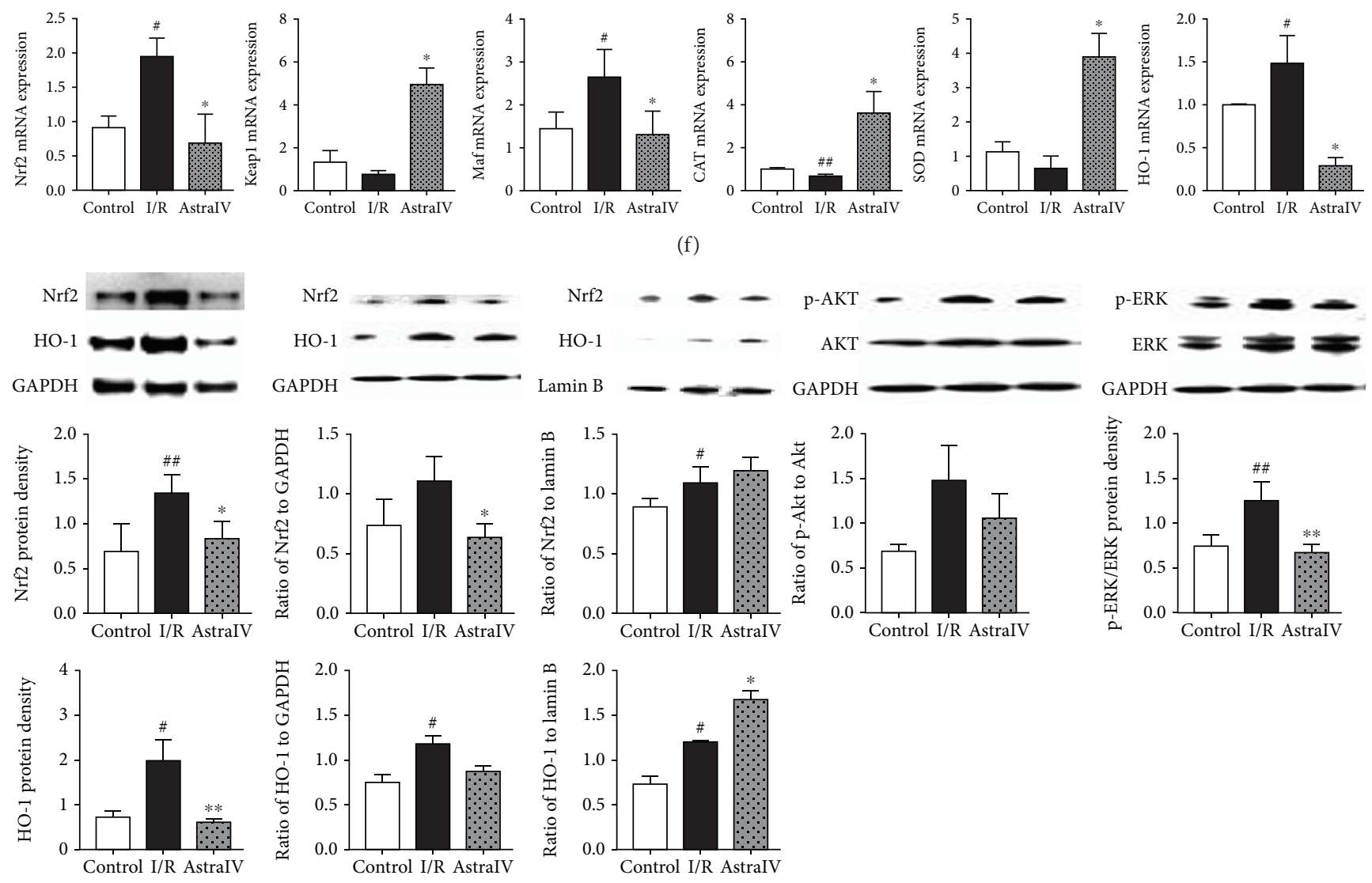

(g)

Figure 1

nitrogen and redissolved in $200 \mu \mathrm{l} \mathrm{D}_{2} \mathrm{O}$ containing $1 \mathrm{mM}$ TSP- $d_{4}$. After centrifugation, $150 \mu \mathrm{l}$ supernatant was transferred into $3 \mathrm{~mm}$ NMR tube for testing. All NMR spectra were recorded at $298 \mathrm{~K}$ on a Bruker AVIII HD $600 \mathrm{MHz}$ spectrometer (Bruker Company, Switzerland) operating at $600.25 \mathrm{MHz}$ for proton resonance frequency. Onedimensional ${ }^{1} \mathrm{H}$ NMR spectra of heart extracts were acquired with CPMGPR1D pulse sequences (from the Bruker pulse sequence library) with water signal presaturation. The time of relaxation delay was set to $4 \mathrm{~s}$, and the $90^{\circ}$ pulse length was adjusted to about $11.65 \mu$ s. Sixty-four transients were recorded into $32 \mathrm{k}$ data points with spectral width of $12019.2 \mathrm{~Hz}$.

Free induction decays (FID) were transformed using MestReNova 6.1.0 (Mestrelab Research S.L., Spain) and then phase- and baseline-corrected manually. The ${ }^{1} \mathrm{H}$ NMR spectra were referenced to the methyl resonance of TSP- $d_{4}(\delta$ $0.000)$ and bucketed into bins of $0.004 \mathrm{ppm}$ in the range of $\delta$ 0.8-10.0 ppm. The regions of $\delta 4.700-5.116$ were removed to eliminate the interference of imperfect water-saturation. All integrated bins were normalized to the peak area at $\delta$ 0.000 of each sample prior to statistical data analysis.

2.5. Lipidomic Profiling Based on UHPLC-MS Analysis. Three milligrams of heart powder were weighed and blended into $500 \mu \mathrm{l}$ water. The mixtures were homogenized via a TissueLyser-48 (Jingxin, Shanghai, China) at $60 \mathrm{~Hz}$ for $90 \mathrm{~s}$.
The homogenate was transferred into a $5 \mathrm{ml}$ glass centrifuge tube and extracted with dichloromethane/methanol $(2: 1, v / v$ ) twice based on the Cequier-Sánchez method [24]. Briefly, $490 \mu \mathrm{l} \mathrm{MeOH}$ and $10 \mu \mathrm{l} \mathrm{MeOH}$ containing $20 \mu \mathrm{g} / \mathrm{ml}$ lysophosphatidylcholine $(12: 0)$ and $20 \mu \mathrm{g} / \mathrm{ml}$ phosphatidylcholine $(11: 0 / 11: 0)$ were added into the homogenate, and then $1 \mathrm{ml}$ dichloromethane was added. Phase separation between aqueous and organic layers was performed by centrifugation at $3000 \mathrm{rpm}$ for $15 \mathrm{~min}$ at room temperature. The organic phase at the bottom was collected into a new 5 $\mathrm{ml}$ glass centrifuge tube. The combined extractions were dried by vacuum, and the resultant powder was dissolved in $200 \mu \mathrm{l}$ isopropanol/methanol $(1: 1, v / v)$ and stored at $-20^{\circ} \mathrm{C}$.

Lipidomic analyses were conducted on an Ultimate ${ }^{\circledR} 3000$ ultra-high-performance liquid chromatography (UHPLC) system coupled to a Q Exactive Hybrid QuadrupoleOrbitrap MS system (Thermo Scientific, MA, USA). Separation was performed on a Hypersil GOLD $\mathrm{C}_{18}(100 \times 2.1 \mathrm{~mm}$, $1.9 \mu \mathrm{m}$, Thermo Scientific, MA, USA), and $4 \mu \mathrm{l}$ of each sample was injected. The flow rate of the mobile phase and the temperature of the column oven were set to $0.35 \mathrm{ml} / \mathrm{min}$ and $45^{\circ} \mathrm{C}$, respectively. The mobile phase comprised acetonitrile/water $(3: 2,10 \mathrm{mM}$ ammonium formate and $0.1 \%$ formic acid, phase A) and acetonitrile/isopropanol (1:9, 10 $\mathrm{mM}$ ammonium formate and $0.1 \%$ formic acid, phase B) following an optimal gradient: $0-14.5 \mathrm{~min}, 40 \%-100 \% \mathrm{~B}$. All the samples were kept at $15^{\circ} \mathrm{C}$ during analyses. The MS raw data 
were acquired using the software Xcalibur (version 3.0, Thermo Scientific). LipidSearch (version 4.0, Thermo Scientific) was used for lipid identification and quantification according to the exact mass, retention time, and the pattern of precursor ions and $\mathrm{MS}^{2}$.

2.6. In Vivo Rat Myocardial Ischemia and Reperfusion. A total of 24 rats were randomly divided into three groups before ischemia-reperfusion (I/R) surgery. The rats in the treated group were administrated intragastrically with astragaloside IV at a dose of $40 \mathrm{mg} / \mathrm{kg}$ once daily for 7 days, while the rats in the sham and $\mathrm{I} / \mathrm{R}$ groups received $10 \mathrm{ml} / \mathrm{kg}$ saline at the same time. After preadministration, rats were anesthetized with $5 \%$ chloral hydrate $(6 \mathrm{ml} / \mathrm{kg})$ and the chest was then opened. The proximal left anterior descending coronary artery (LADCA) was ligated with a 6/0 silk, which was released after $30 \mathrm{~min}$ allowing reperfusion to occur. The thorax was subsequently closed, and as soon as spontaneous respiration was sufficient, the rats were released and allowed to recover on an electric blanket. The animals in the sham group underwent the same procedure but without ligation on LADCA. A Vevo 2100 ultra-high-resolution small animal ultrasound imaging system (VisualSonics Vevo 2100, Canada) with a MS-250 ultrasound scanning transducer (model C5) was employed to acquire the parameters of the left ventricular function in real time, including the left ventricle ejection fraction percentage (EF\%), aortic valve peak velocity (AV peak), $+\mathrm{d} p / \mathrm{d} t_{\max },-\mathrm{d} p / \mathrm{d} t_{\max }$, and left ventricle fractional shortening percentage (FS\%). At the end of the experiments, all data were analyzed off-line using the resident software in the ultrasound system. The hematoxylin-eosin (HE) stain was performed on the myocardium sections, and the levels of $\mathrm{CK}$ and $\mathrm{LDH}$ in the serum were determined by commercial kits.

2.7. Quantitative Real-Time PCR. Total RNA was extracted from myocardial tissue using a TRIzol reagent (Invitrogen, Carlsbad, CA), and DNase-treated RNA was reverse transcribed with the use of a Transcriptor First-Strand cDNA Synthesis Kit (Roche, Indianapolis, IN). Quantitative realtime PCR analysis was done in triplicate using the FastStart Universal SYBR Green Master (Roche). PCR primers were synthesized from Sangon Biotech Co. Ltd. (Shanghai, China) as follows: for superoxide dismutase (SOD), AGATGACTT GGGCAAAGGTG and CAATCCCAATCACACCACAA; for nuclear factor (erythroid-derived 2)-like 2 (Nrf2), TTCC TCTGCTGCCATTAGTCAGTC and GCTCTTCCATT TCCGAGTCACTG; for heme oxygenase-1 (HO-1), CACG CATATACCCGCTACCT and CCAGAGTGTTCATTCG AGCA; for catalases (CAT), ACATGGTCTGGGACTT CTGG and CCATTCGCATTAACCAGCTT; for Kelch-like ECH-associated protein 1 (Keap-1), AGCAGATCGGCTGC ACTGAA and AGCTGGCAGTGTGACAGGTTG; for musculoaponeurotic fibrosarcoma (Maf), AAGGAGGAGGT GATCCGACT and TCGAGCAGTTTTCTCGGAAC; and for glyceraldehyde-3-phosphate dehydrogenase (GAPDH), ATGATTCTACCCACGGCAAG and CTGGAAGATGG TGATGGGTT. Among them, GAPDH was employed as a housekeeping gene. Relative mRNA expression levels were calculated by the $2^{-\Delta \Delta \mathrm{Ct}}$ method.

2.8. Western Blot Analysis. After treatment and washing with cold PBS, myocardial tissue cut from the left ventricle surrounding (30 $\mathrm{mg}$ ) was lysed in a radioimmunoprecipitation assay lysis buffer (Sangon Biotech Co. Ltd., Shanghai, China) to extract the whole protein. Tissue lysate was suspended in $400 \mu \mathrm{l}$ of cold buffer A (10 mM HEPES, pH 7.9, $10 \mathrm{mM}$ $\mathrm{KCl}, 0.1 \mathrm{mM}$ EDTA, $0.1 \mathrm{mM}$ EGTA, $1 \mathrm{mM}$ DTT, and 0.5 $\mathrm{mM}$ PMSF) and incubated for $15 \mathrm{~min}$ on ice followed by the addition of $50 \mu \mathrm{l}$ of $10 \%$ Nonidet P-40 and vortex for $10 \mathrm{~s}$. After spinning for $30 \mathrm{~s}$ at $16,200 \times \mathrm{g}$ at $4^{\circ} \mathrm{C}$, the pellet was saved and resuspended in $100 \mu \mathrm{l}$ of cold buffer $\mathrm{C}(20$ mM HEPES, pH 7.91,400 mM KCl, $1 \mathrm{mM}$ EDTA, $1 \mathrm{mM}$ EGTA, 1 mM DTT, 1 mM PMSF, and $10 \mu \mathrm{g} / \mathrm{ml}$ leupeptin/aprotinin) and kept on ice for $15 \mathrm{~min}$. The mixture was spun again for $5 \mathrm{~min}$, and the supernatant was collected as nuclear proteins and kept at $-80^{\circ} \mathrm{C}$ until assay. The concentrations of the total protein in myocardial tissues and nuclei were determined by bicinchoninic acid (BCA) protein assay kit (Thermo Fisher Scientific, Waltham, USA). Protein samples were separated on SDS-PAGE gels and transferred to polyvinylidene difluoride membrane (Millipore, Bedford, MA). The membranes were probed with various primary antibodies overnight at $4^{\circ} \mathrm{C}$. After incubation with secondary antibodies, the membranes were treated with ECL reagents. Protein levels were quantified with a VersaDoc MP 5000 multifunctional imaging analysis system (Bio-Rad Laboratories Inc., Hercules, CA, USA). Primary antibodies against protein kinase B (Akt, 4691, dilution ratio 1:500), phosphorylation Akt (p-Akt, 4060, dilution ratio 1:500), extracellular signal-regulated kinase $1 / 2(\mathrm{ERK} 1 / 2,4695$, dilution ratio 1:500), phosphorylation ERK1/2 (p-ERK1/2, 4370, dilution ratio $1: 500), \mathrm{HO}-1$ (82206, dilution ratio $1: 1000), \mathrm{GAPDH}$ (5174, dilution ratio $1: 1000$ ), and HRP-linked goat anti-rabbit/rat IgG secondary antibody (7074, dilution ratio $1: 1000)$ were purchased from Cell Signaling Technology Inc. (MA, USA). The primary antibody against Nrf2 (sc-722) was purchased from Santa Cruz Biotechnology Inc. (CA, USA).

2.9. Statistical Analysis. Data were expressed as mean \pm s.d., and $p$ values were calculated using the two-tailed Student's $t$-test for pairwise comparisons and the one-way analysis of variance (ANOVA) for multiple comparisons. Comparisons between multiple-group means were performed using oneway analysis of variance (one-way ANOVA). Principal component analysis (PCA) and orthogonal partial least squaresdiscriminant analysis (OPLS-DA) were performed on SIMCA-P 14.1 software (Umetrics, Umea, Sweden).

\section{Results}

3.1. Astragaloside IV Prevents the Progress of Ischemia of Reperfusion Injury on the Heart. We first assessed the cardioprotective effect of astragaloside IV using the ex vivo (EV) Langendorff heart experiment. Twenty minutes of ischemia and 30 minutes of reperfusion resulted in myocardial injury in $\mathrm{EV}$ hearts, as evidenced by increasing infarct size $(p<0.01$, 

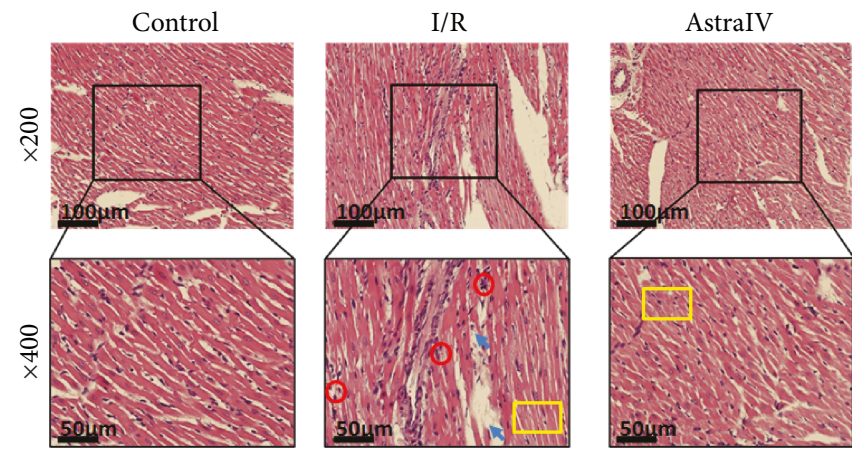

(a)
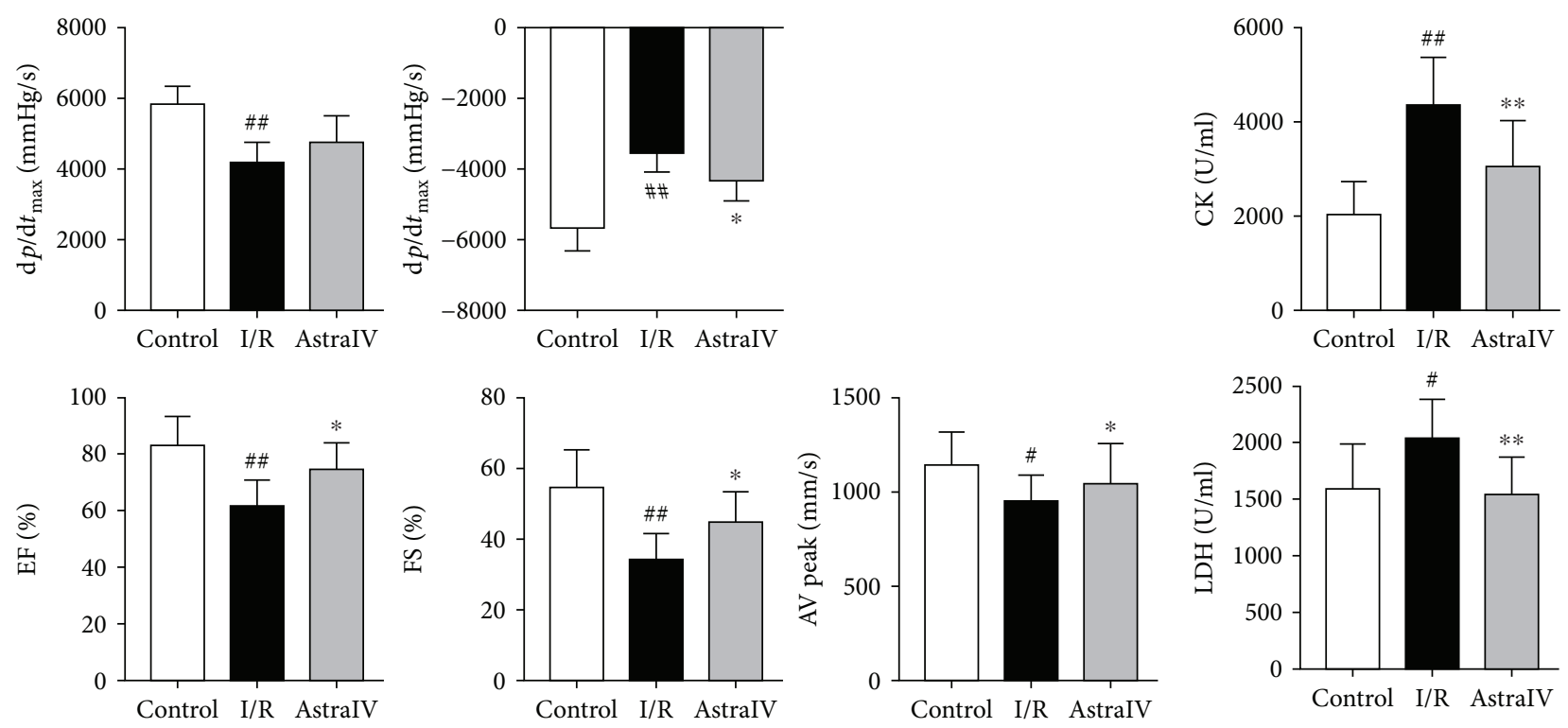

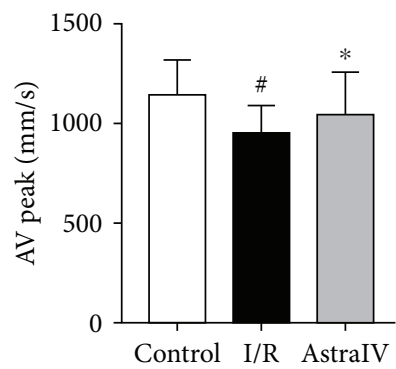

(b)

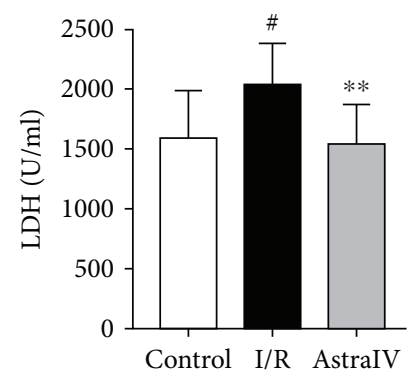

(c)

Figure 2: Continued.

Figure $1(\mathrm{~b}))$, CK $(p<0.05)$, and LDH activities $(p<0.01$, Figure $1(\mathrm{c})$ ), along with decreasing cardiac function parameters, including HR, LVDP, and $\pm \mathrm{d} p / \mathrm{d} t_{\max }(p<0.01$, Figure $1(\mathrm{~d}))$. Astragaloside IV pretreatment significantly reduced infarct size $(p<0.01)$, CK $(p<0.01)$, and LDH $(p<0.05)$ releases in the EV hearts of the drug-treated group and also elevated systolic and diastolic function parameters $(p<0.01)$ compared with those in the corresponding I/R model group.

We next employed the in vivo (IV) rat model of myocardial ischemia and reperfusion to validate the efficiency of astragaloside IV. According to HE staining images (Figure 2(a)), myocardial edema, rupture of myocardial fibers, and infiltration of leukocytes were observed in the surrounding areas of infarction in the IV hearts of the I/R group, which were obviously prevented by using astragaloside IV in advance. Moreover, increase of $\mathrm{EF} \%, \mathrm{FS} \%, \mathrm{AV}$ peak, and $\pm \mathrm{d} p / \mathrm{d} t_{\max }$ indicated that the cardiac function was significantly improved in the drug-treated group compared with the I/R group ( $p<0.05$, Figure 2(b)). Astragaloside IV also decreased the releases of CK and LDH in the serum $(p<0.01$, Figure $2(c))$. These results revealed that astragaloside IV protected both the EV and IV rat hearts against I/R injury.
3.2. Astragaloside IV Suppressed Cardiac Oxidation Stress by the Induction of Nrf2 Expression. Oxidative stress plays an important role in the ischemic cascade due to oxygen reperfusion injury following hypoxia. Since MDA is a lipid peroxidation marker of oxidative stress, we assessed its levels in EV rat hearts. As shown in Figure 1(e), astragaloside IV pretreatment significantly suppressed MDA levels $(p<0.05)$ and induced the activities of the antioxidant enzymes, SOD, and SDH $(p<0.01)$, in the myocardial tissues compared with the I/R group.

The transcription factor Nrf2 is considered to be one of the most important cellular defense mechanisms against oxidative stress with a particular role in the regulation of phase II detoxifying enzymes and antioxidant status. Its activation has been found to be primarily controlled by Keap 1 . Upstream kinases (e.g., Akt and ERK) activate Nrf2 through phosphorylation at specific sites favoring the release of Nrf2 from Keap1. Many endogenous enzymes (e.g., SOD, CAT, and $\mathrm{HO}-1$ ) can be subsequently upregulated through the binding of Nrf2 to the antioxidant response element found in the promoters of these genes. We first quantified the expression of Keap1, Nrf2, HO-1, SOD, Maf, and CAT 

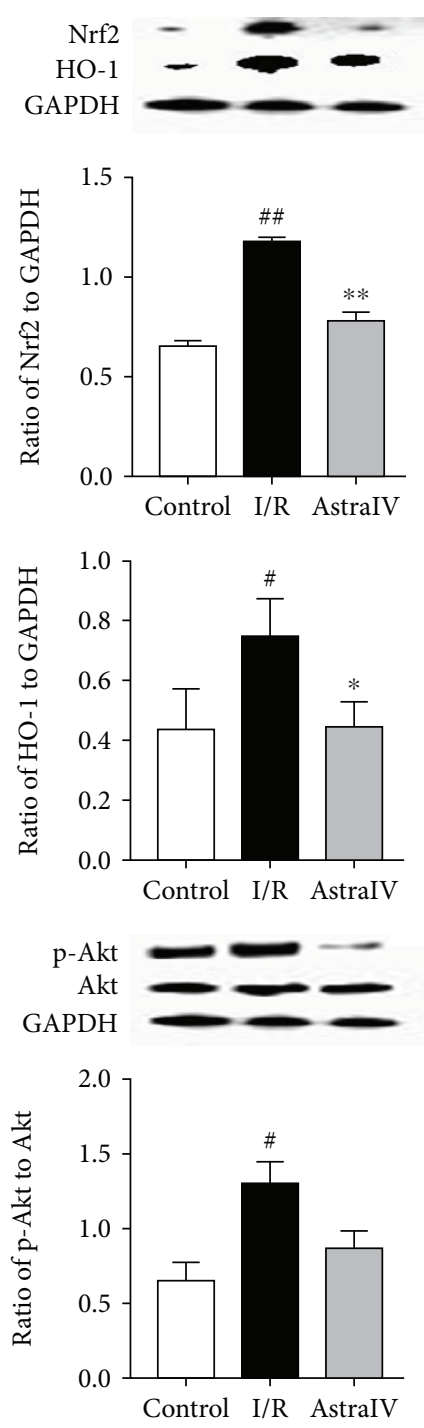
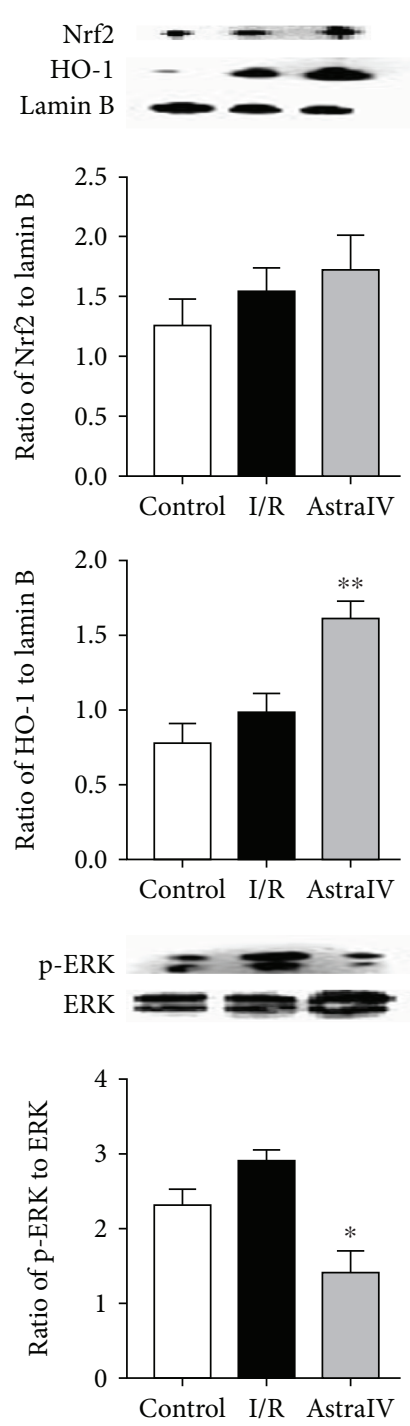

(d)

FIGURE 2

mRNA in EV hearts by qRT-PCR technology (Figure 1(f)). The results indicated that astragaloside IV pretreatment increased Keap1 $(p<0.05)$, SOD $(p<0.05)$, and CAT mRNA expressions and decreased Nrf2 $(p<0.05), \mathrm{HO}-1(p<0.05)$, and Maf $(p<0.05)$ mRNA expressions compared with the I/R group.

We further measured the expression of Nrf2, HO-1, pAkt, Akt, p-ERK, and ERK proteins in EV and IV hearts by the immunoblotting method (Figure 1(g) and Figure 2(d)). Compared to the sham-operated group, I/R injury stimulated Nrf2 and HO-1 protein expression in both cardiomyocytes and the nucleus. The ratios of p-Akt to Akt and p-ERK1/2 to ERK1/2 were also increased in the I/R group samples. Via preconditioning before $\mathrm{I} / \mathrm{R}$, astragaloside IV inhibited the total protein expression of Nrf2 and HO- 1 in cardiomyocytes and decreased the ratios of p-Akt to Akt and p-ERK1/2 to ERK1/2, while promoting the protein levels of $\mathrm{Nrf} 2$ and HO- 1 in the nucleus compared with the I/R group. It indicated that astragaloside IV probably activated the
Nrf2/HO-1 pathway to exert antioxidant stress during the process of I/R injury.

3.3. Astragaloside IV-Adjusted I/R Injury Induced Metabolic Changes. The signal resonances in ${ }^{1} \mathrm{H}$ NMR spectra of EV heart extracts from the control, I/R, and drug-treated groups were elucidated and assigned on the basis of chemical shift values and coupling constants and further confirmed by literature data and a range of $2 \mathrm{D}-\mathrm{NMR}$ experiments (Table 1 ). Forty-six metabolites were identified in the extracts including amino acids, carbohydrates (glucose, mannose, and sucrose), TCA cycle intermediate (succinate), organic acid (lactate, 2phenylpropionate), ketone bodies (3-hydroxybutyrate (3$\mathrm{HB})$ ), purine and pyrimidine metabolites, nicotinurate, creatine, and choline metabolites.

Multivariate analyses were conducted thoroughly for the NMR data obtained from myocardium tissue extracts. To identify the metabolites that significantly contributed to grouping, we performed OPLS-DA on normalized and unit- 


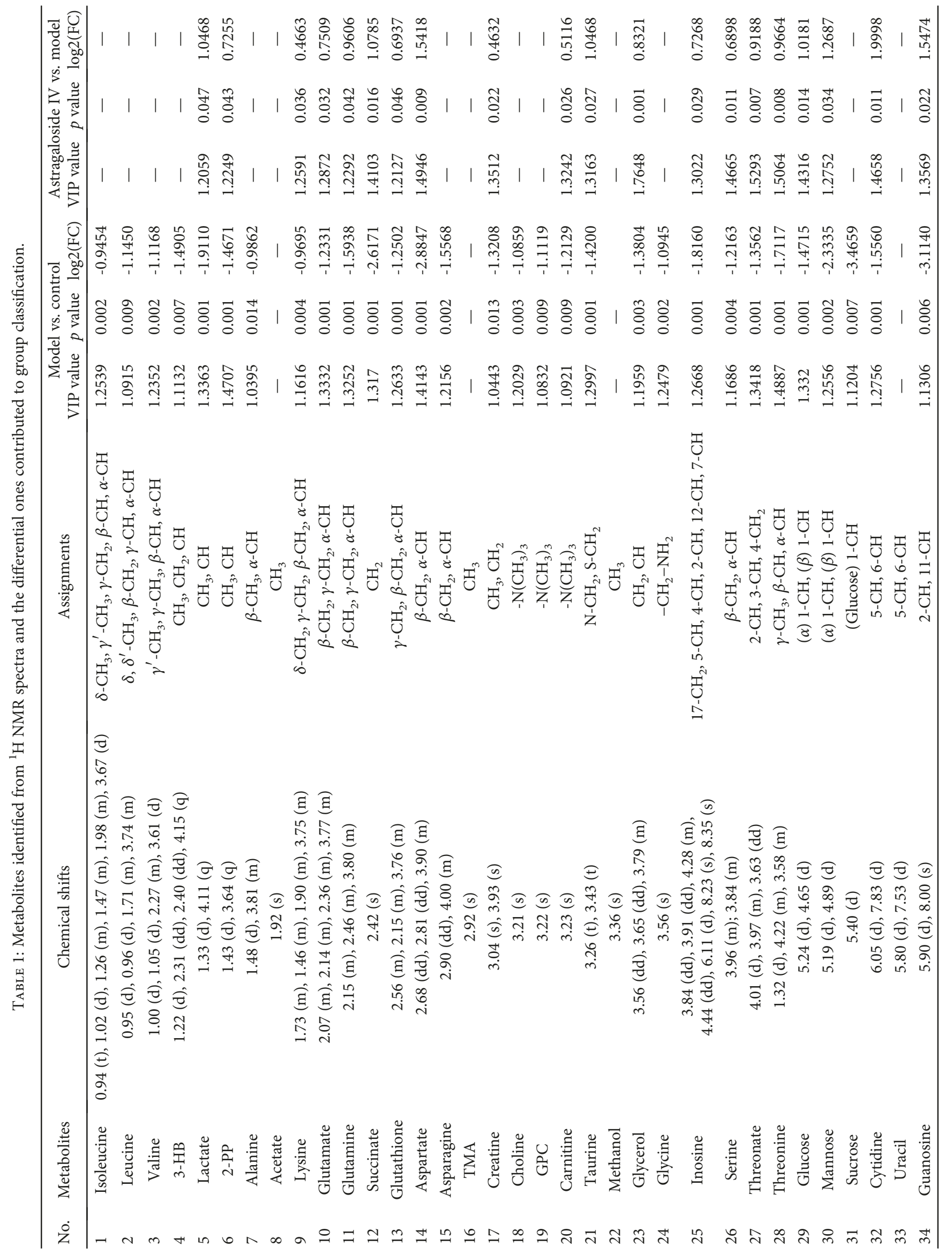




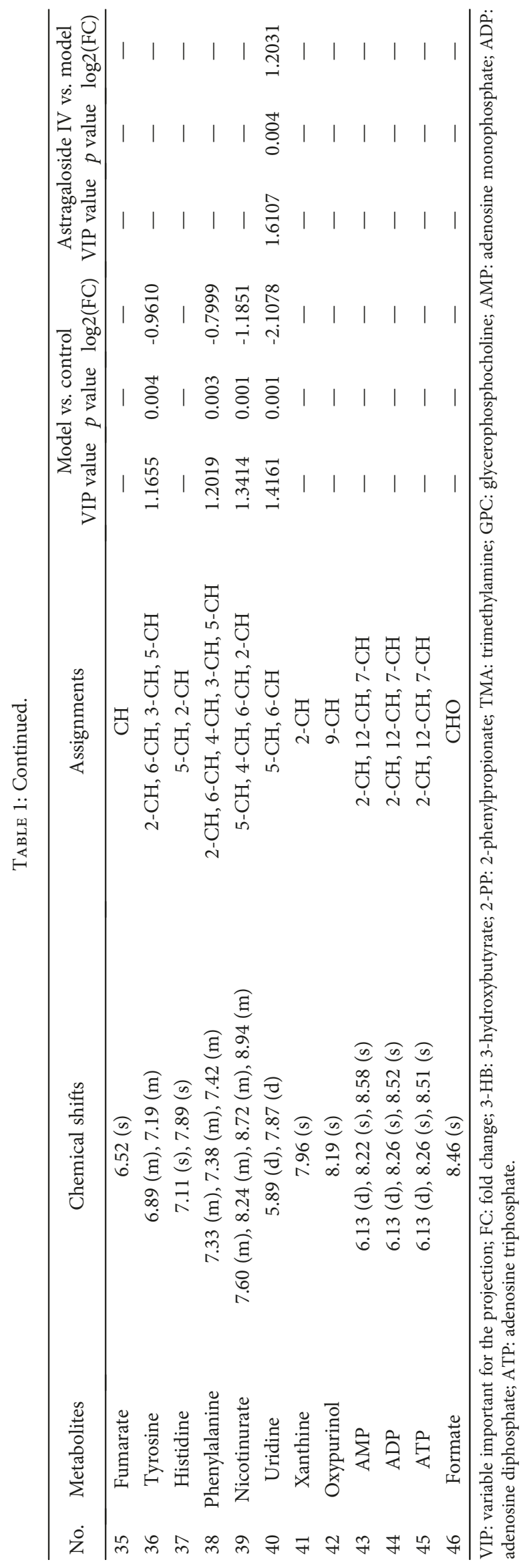



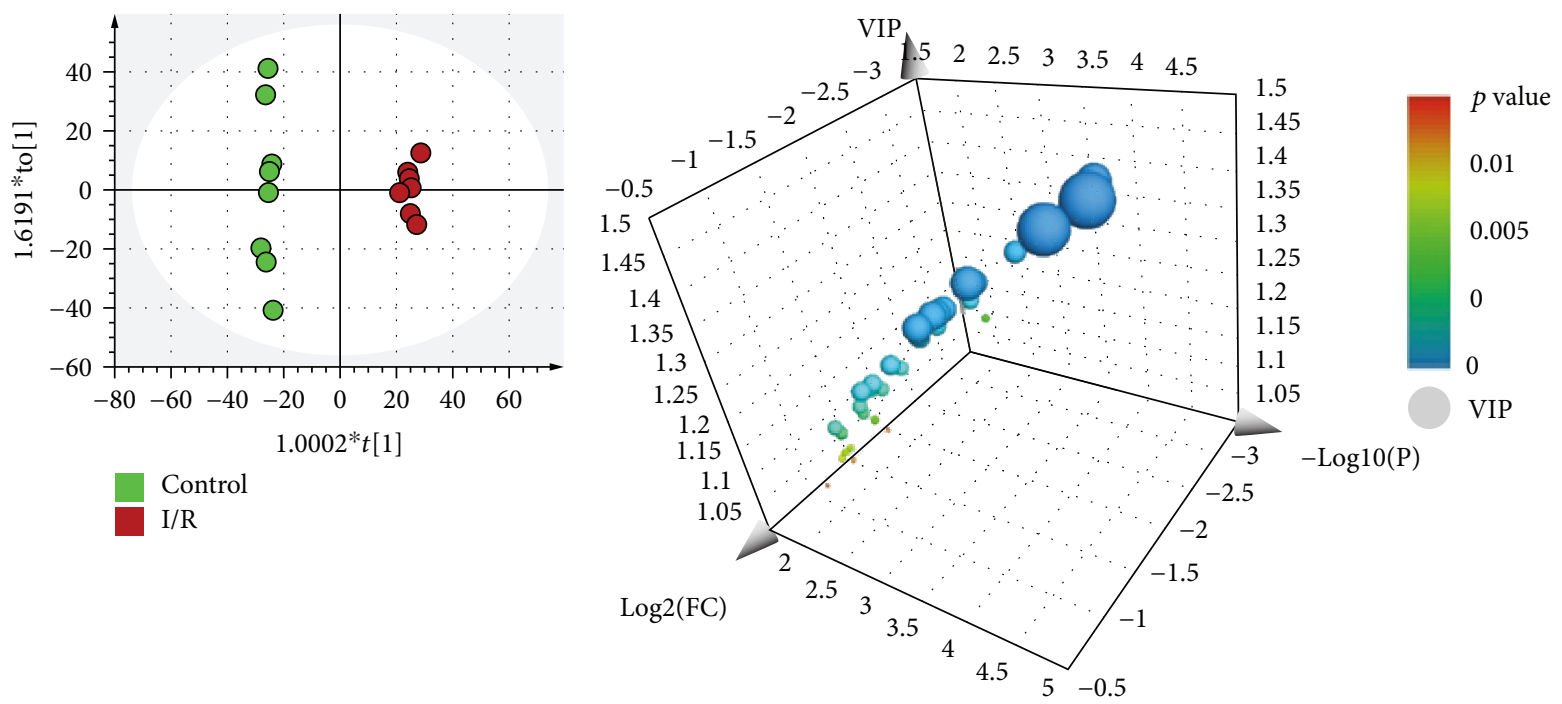

(a)
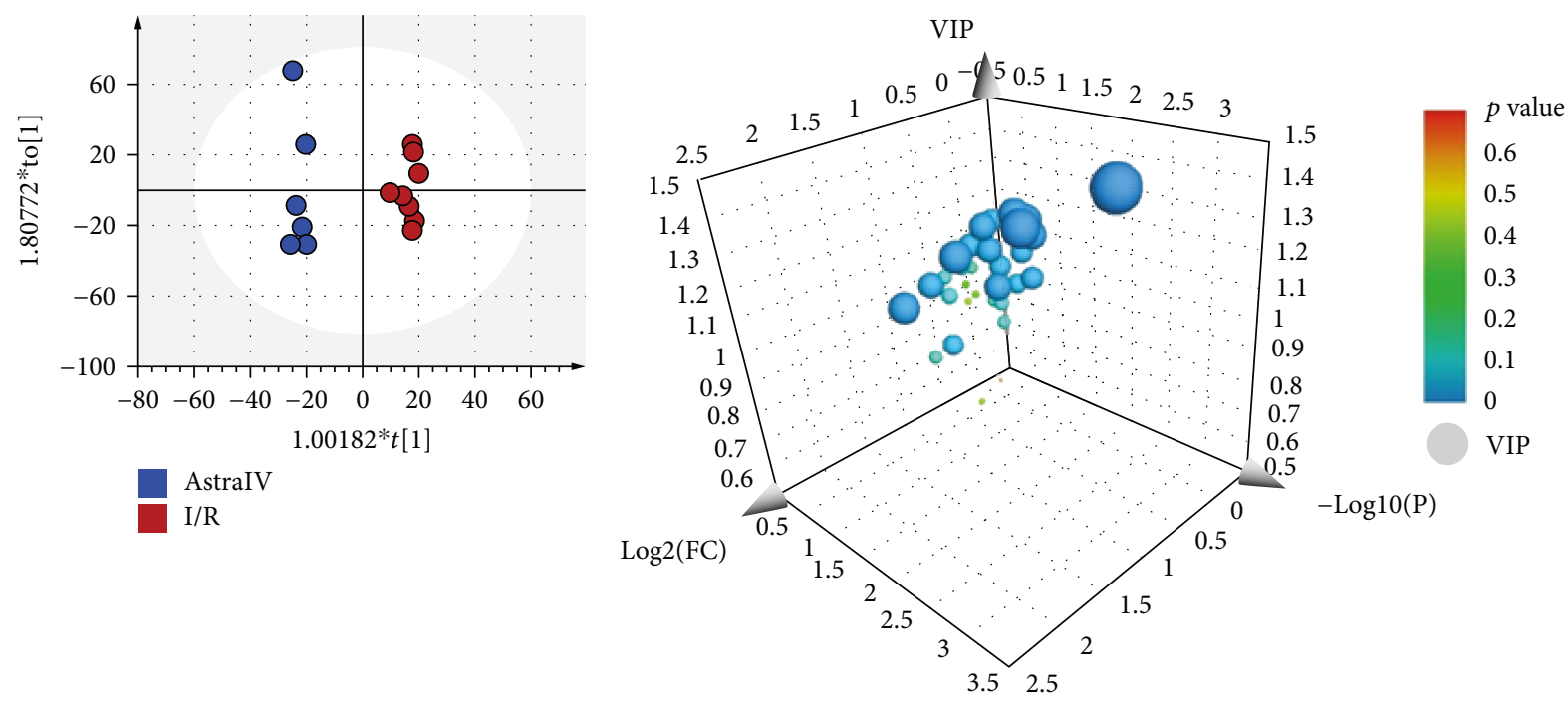

(c)

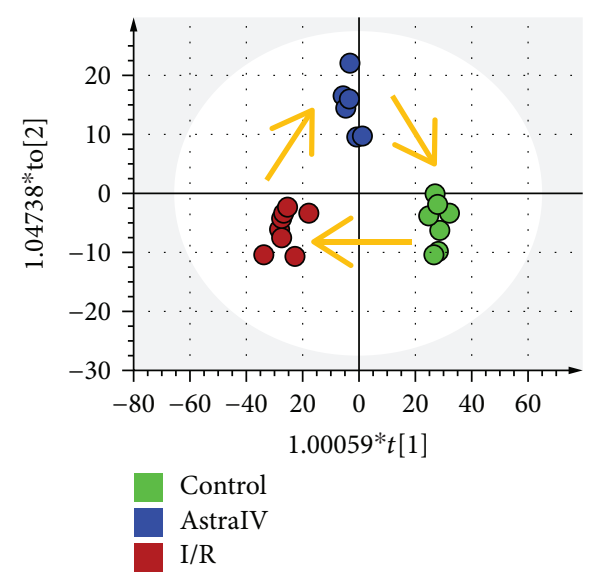

(d)

(e)

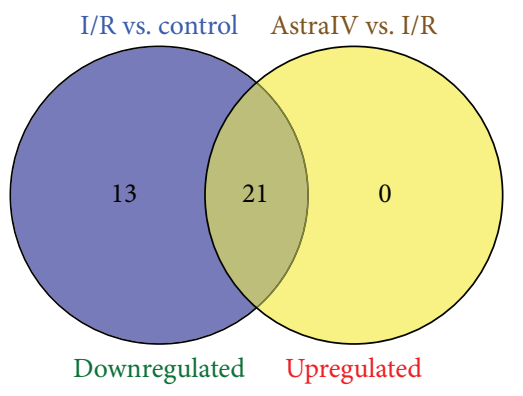

(f)

Figure 3 


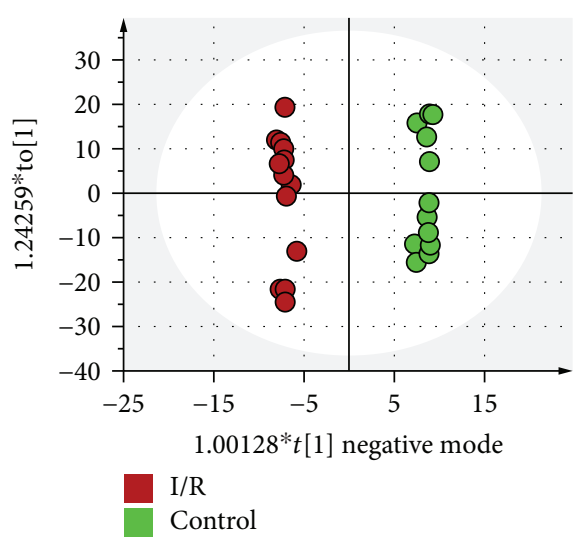

(a)

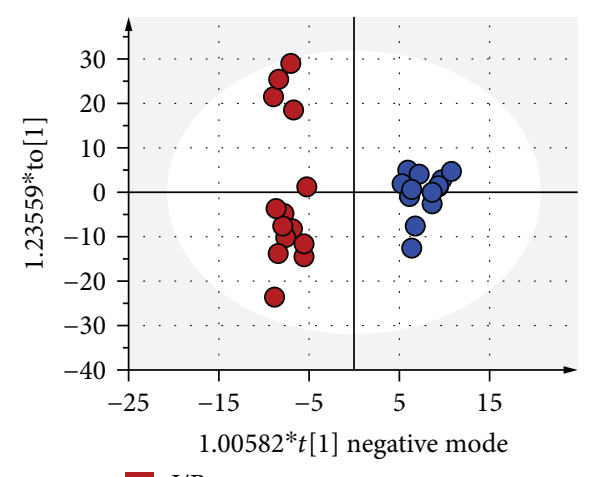

$\mathrm{I} / \mathrm{R}$

AstraIV

(c)

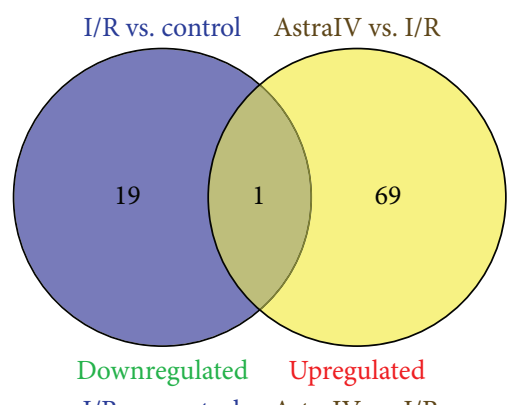

I/R vs. control AstraIV vs. I/R

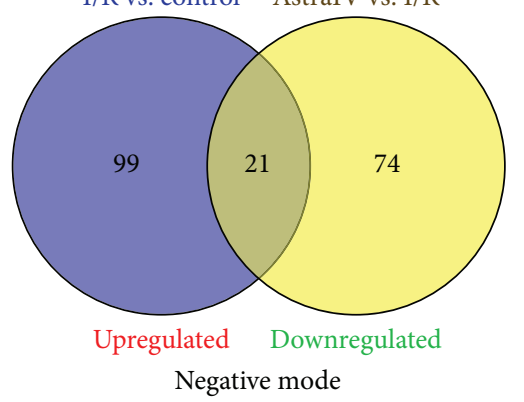

(e)

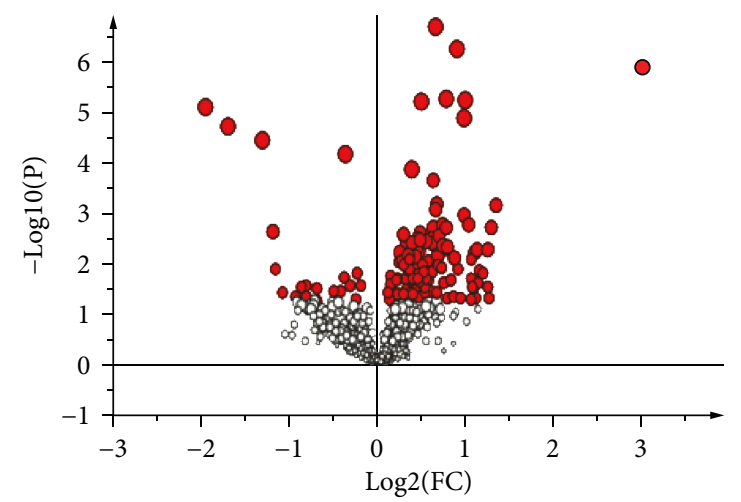

VIP

- 0.8

- 1.6

- 2.2

(b)

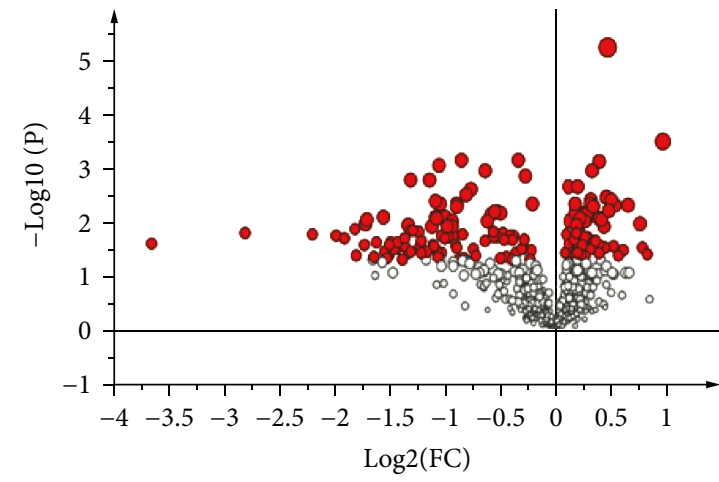

VIP

- 0.8

- 1.6

- 2.2

(d)

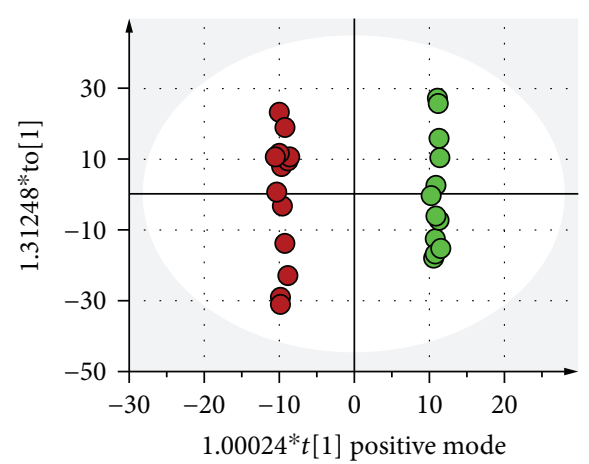

$\mathrm{I} / \mathrm{R}$

Control

FIgUre 4: Continued. 


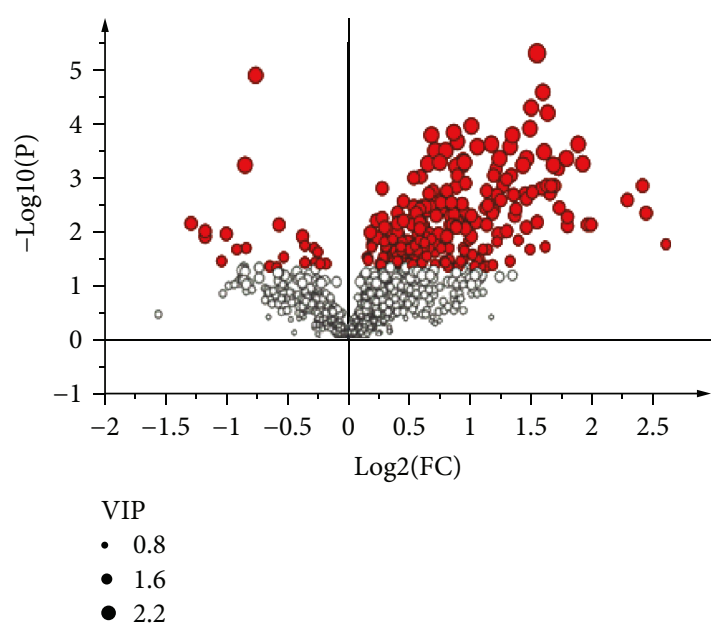

(g)

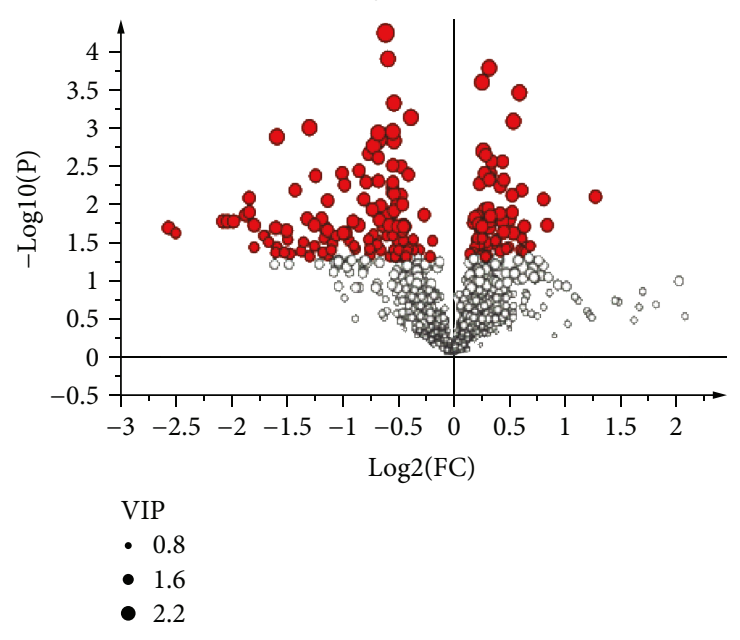

(i)

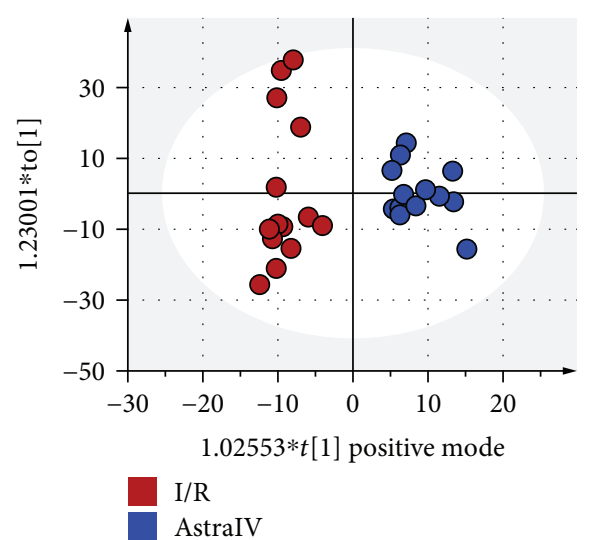

(h)

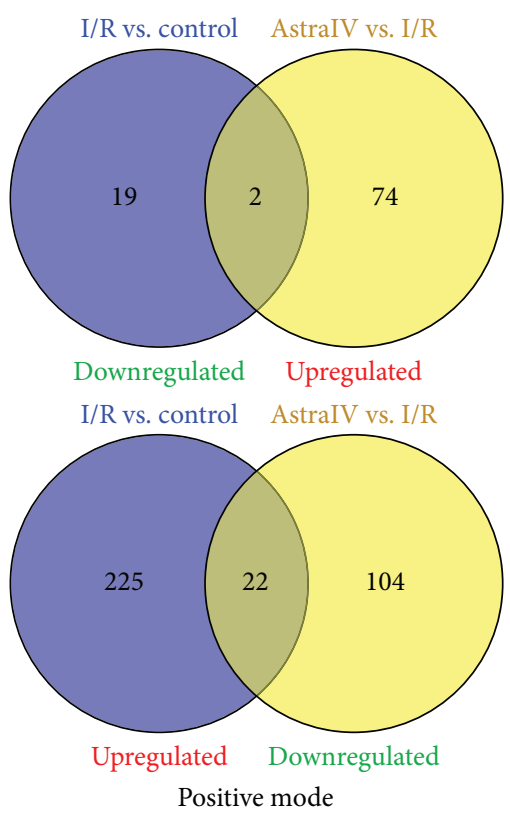

(j)

Figure 4

variance (UV) scaling data. The score plots from OPLS-DA showed significant metabolomic differences between control and I/R groups $\left(R^{2} X=0.672, R^{2} Y=0.996, Q^{2}=0.778\right.$; Figure 3(a)), as well as between $\mathrm{I} / \mathrm{R}$ and drug-treated groups $\left(R^{2} X=0.657, R^{2} Y=0.981, Q^{2}=0.538\right.$; Figure 3(c)). With the screening conditions of VIP $>1$ and $p<0.05,34$ metabolites were extracted and identified as potential biomarkers to distinguish the I/R group from the control group (Figure 3(b), Table 1), all of which were downregulated after I/R injury. Among them, 21 metabolites suggested significant intergroup differences between the I/R group and the drug-treated group and were upregulated with the pretreatment of astragaloside IV (Figure 3(d)-3(f), Table 1).

3.4. Astragaloside IV-reduced I/R Injury Induced Lipidomic Changes. Lipid species from cardiolipin (CL), ceramide (Cer), fatty acids (FA), digalactosyl diacylglycerol (DGDG), lysopho- sphatidylcholine (LPC), lysophosphatidylethanolamine (LPE), lysophosphatidylglycerol (LPG), lysophosphatidylinositol (LPI), lysophosphatidylserine (LPS), monogalactosyl diacylglycerol (MGDG), (O-acyl)-hydroxy fatty acids (OAHFA), phosphatidic acid (PA), phosphatidylcholine (PC), phosphatidylethanolamine (PE), phosphatidylglycerol (PG), phosphatidylinositol (PI), phosphatidylserine (PS), sphingomyelin (SM), sphingosine (So), coenzyme (Co), diacylglycerol (DG), monoacylglycerol (MG), and triacylglycerol (TG) lipid categories were identified from the EV heart extracts, and the PCA score plots suggested that it was not necessary to remove any outlier of the data. Detailed lipidomic changes were obtained by constructing OPLS-DA models with the fitness and predictability expressed by the values of $R^{2}$ and $Q^{2}$. Differentiate ions in each pairwise comparison were selected by VIP value $>1$ and $p<0.05$. The score plots from OPLS-DA displayed a clear separation between the control and I/R groups in the negative 
Table 2: Differential lipid species contributed to group classification.

\begin{tabular}{|c|c|c|c|c|c|c|c|c|}
\hline \multirow{2}{*}{ No. } & \multirow{2}{*}{ Lipid } & \multirow{2}{*}{ Lipid ion } & \multicolumn{3}{|c|}{ Model vs. control } & \multicolumn{3}{|c|}{ Astragaloside IV vs. model } \\
\hline & & & VIP value & $p$ value & $\log 2(\mathrm{FC})$ & VIP value & $p$ value & $\log 2(\mathrm{FC})$ \\
\hline 1 & DG $(18: 1 / 22: 4)$ & DG $(18: 1 / 22: 4)+\mathrm{NH} 4$ & 2.2076 & 0.001 & 1.6410 & 1.4538 & 0.040 & -0.6451 \\
\hline 2 & CL $(18: 2 / 18: 2 / 20: 3 / 18: 2)$ & CL $(18: 2 / 18: 2 / 20: 3 / 18: 2)-\mathrm{H}$ & 2.5225 & 0.001 & 0.4739 & 1.4087 & 0.035 & -0.2150 \\
\hline 3 & DG $(22: 6 / 22: 6)$ & DG $(22: 6 / 22: 6)+\mathrm{NH} 4$ & 1.2593 & 0.008 & -1.2863 & 1.4958 & 0.008 & 1.2987 \\
\hline 4 & FA $(24: 5)$ & FA $(24: 5)-\mathrm{H}$ & 1.8095 & 0.004 & 0.6340 & 1.5863 & 0.016 & -0.4651 \\
\hline 5 & $\operatorname{LPC}(15: 0)$ & $\operatorname{LPC}(15: 0)+\mathrm{H}$ & 1.4051 & 0.023 & 0.5568 & 1.7333 & 0.013 & -0.5419 \\
\hline \multirow[t]{2}{*}{6} & $\operatorname{LPC}(16: 0)$ & $\operatorname{LPC}(16: 0)+\mathrm{H}$ & 1.4575 & 0.017 & 0.4393 & 1.8399 & 0.008 & -0.4683 \\
\hline & & LPC $(16: 0)+\mathrm{HCOO}$ & 1.8306 & 0.003 & 0.4709 & 1.4347 & 0.031 & -0.2877 \\
\hline 7 & LPC $(16: 1 p)$ & $\operatorname{LPC}(16: 1 p)+\mathrm{H}$ & 1.6036 & 0.008 & 0.5349 & 1.8856 & 0.006 & -0.5417 \\
\hline \multirow[t]{2}{*}{8} & LPC $(17: 0)$ & $\operatorname{LPC}(17: 0)+\mathrm{H}$ & 1.8525 & 0.007 & -0.5395 & 1.4751 & 0.016 & 0.4943 \\
\hline & & LPC $(17: 0)+\mathrm{HCOO}$ & 2.0689 & 0.001 & 0.6604 & 1.5609 & 0.018 & -0.3770 \\
\hline 9 & $\operatorname{LPC}(18: 0)$ & $\operatorname{LPC}(18: 0)+\mathrm{H}$ & 1.5194 & 0.013 & 0.4179 & 1.5409 & 0.029 & -0.3576 \\
\hline \multirow[t]{2}{*}{10} & $\operatorname{LPC}(18: 1)$ & $\operatorname{LPC}(18: 1)+\mathrm{H}$ & 1.3712 & 0.026 & 0.7205 & 1.8124 & 0.009 & -0.7992 \\
\hline & & LPC $(18: 1)+\mathrm{HCOO}$ & 2.0410 & 0.001 & 0.6449 & 1.5586 & 0.018 & -0.4051 \\
\hline 11 & LPC (18:1p) & $\mathrm{LPC}(18: 1 \mathrm{p})+\mathrm{H}$ & 1.6013 & 0.008 & 0.6067 & 1.6208 & 0.021 & -0.4767 \\
\hline 12 & $\operatorname{LPC}(20: 1)$ & LPC $(20: 1)+\mathrm{HCOO}$ & 1.5674 & 0.014 & 0.4916 & 1.7422 & 0.007 & -0.5742 \\
\hline 13 & $\operatorname{LPC}(20: 3)$ & $\operatorname{LPC}(20: 3)+\mathrm{H}$ & 1.5281 & 0.012 & 0.7497 & 1.7114 & 0.014 & -0.5899 \\
\hline \multirow[t]{2}{*}{14} & $\operatorname{LPC}(20: 4)$ & $\operatorname{LPC}(20: 4)+\mathrm{H}$ & 1.7039 & 0.004 & 0.6532 & 1.6392 & 0.019 & -0.4448 \\
\hline & & LPC $(20: 4)+\mathrm{HCOO}$ & 1.8538 & 0.003 & 0.6563 & 1.5171 & 0.022 & -0.3879 \\
\hline 15 & $\operatorname{LPC}(22: 4)$ & $\operatorname{LPC}(22: 4)+\mathrm{H}$ & 1.7633 & 0.003 & 0.7929 & 2.0677 & 0.002 & -0.7530 \\
\hline \multirow[t]{2}{*}{16} & $\operatorname{LPC}(22: 6)$ & $\mathrm{LPC}(22: 6)+\mathrm{H}$ & 1.7244 & 0.004 & 0.6828 & 1.4231 & 0.045 & -0.3728 \\
\hline & & LPC $(22: 6)+\mathrm{HCOO}$ & 1.9491 & 0.002 & 0.7185 & 1.3837 & 0.038 & -0.3549 \\
\hline 17 & LPE $(17: 0)$ & LPE $(17: 0)+\mathrm{H}$ & 1.3783 & 0.026 & 0.3498 & 1.7758 & 0.010 & -0.5035 \\
\hline 18 & LPG $(16: 0)$ & LPG $(16: 0)-\mathrm{H}$ & 1.5975 & 0.012 & 1.1349 & 1.7168 & 0.008 & -1.0794 \\
\hline 19 & LPG $(18: 1)$ & LPG (18:1)-H & 1.3321 & 0.041 & 0.8443 & 1.7138 & 0.008 & -0.9971 \\
\hline 20 & LPI $(18: 1)$ & LPI $(18: 1)-\mathrm{H}$ & 1.3081 & 0.045 & 1.0946 & 1.3901 & 0.037 & -1.0277 \\
\hline 21 & LPI $(18: 2)$ & LPI $(18: 2)-\mathrm{H}$ & 1.4379 & 0.026 & 1.2306 & 1.5986 & 0.015 & -1.2328 \\
\hline 22 & LPI $(20: 4)$ & LPI $(20: 4)-\mathrm{H}$ & 1.4179 & 0.029 & 1.0758 & 1.3296 & 0.047 & -0.8204 \\
\hline 23 & MG $(22: 6)$ & MG $(22: 6)+\mathrm{H}$ & 1.3515 & 0.029 & 0.4772 & 1.6409 & 0.019 & -0.6263 \\
\hline 24 & PC $(16: 2 / 18: 2)$ & $\mathrm{PC}(16: 2 / 18: 2)+\mathrm{HCOO}$ & 1.0640 & 0.042 & -1.0172 & 1.0495 & 0.043 & 0.7557 \\
\hline 25 & PC $(17: 0 / 18: 2)$ & $\mathrm{PC}(17: 0 / 18: 2)+\mathrm{HCOO}$ & 1.4689 & 0.023 & 0.7415 & 2.0816 & 0.001 & -1.0504 \\
\hline 26 & PC $(36: 5)$ & $\mathrm{PC}(36: 5)+\mathrm{H}$ & 2.0056 & 0.001 & 1.8005 & 2.1950 & 0.001 & -1.2854 \\
\hline 27 & PC $(38: 4)$ & $\mathrm{PC}(38: 4)+\mathrm{H}$ & 1.3104 & 0.035 & 0.4454 & 1.7385 & 0.012 & -0.5253 \\
\hline 28 & $\mathrm{PC}(41: 4)$ & $\mathrm{PC}(41: 4)+\mathrm{H}$ & 2.2853 & 0.001 & 1.6044 & 1.9778 & 0.004 & -0.8453 \\
\hline 29 & $\mathrm{PE}(16: 0 / 18: 1)$ & $\mathrm{PE}(16: 0 / 18: 1)-\mathrm{H}$ & 1.7538 & 0.005 & 0.4561 & 1.7541 & 0.007 & -0.4875 \\
\hline 30 & PE $(18: 0 / 20: 5)$ & PE $(18: 0 / 20: 5)-\mathrm{H}$ & 1.2958 & 0.048 & 1.0505 & 1.6508 & 0.012 & -1.3285 \\
\hline 31 & PE $(39: 5)$ & $\mathrm{PE}(39: 5)+\mathrm{H}$ & 1.9500 & 0.001 & 1.7287 & 1.5870 & 0.024 & -0.9653 \\
\hline 32 & PE (19:0e) & $\mathrm{PE}(19: 0 \mathrm{e})+\mathrm{H}$ & 1.4636 & 0.017 & 0.4501 & 1.7815 & 0.010 & -0.4539 \\
\hline 33 & PG $(17: 0 / 18: 1)$ & PG $(17: 0 / 18: 1)-H$ & 1.3876 & 0.033 & 0.1410 & 2.0119 & 0.001 & -0.2670 \\
\hline 34 & PS $(37: 3)$ & PS $(37: 3)-\mathrm{H}$ & 2.6293 & 0.001 & 3.0100 & 1.3271 & 0.048 & -0.4890 \\
\hline 35 & PS $(40: 6)$ & PS $(40: 6)+\mathrm{H}$ & 1.3239 & 0.008 & -0.5606 & 1.1997 & 0.008 & 0.5361 \\
\hline 36 & $\mathrm{SM}(\mathrm{d} 22: 1 / 20: 1)$ & $\mathrm{SM}(\mathrm{d} 22: 1 / 20: 1)+\mathrm{HCOO}$ & 2.6812 & 0.001 & 0.8807 & 1.5137 & 0.022 & -0.2800 \\
\hline 37 & $\mathrm{SM}(\mathrm{d} 39: 1)$ & $\mathrm{SM}(\mathrm{d} 39: 1)+\mathrm{HCOO}$ & 1.8395 & 0.003 & 0.4619 & 1.4322 & 0.031 & -0.3028 \\
\hline 38 & $\mathrm{SM}(\mathrm{d} 42: 1)$ & $\mathrm{SM}(\mathrm{d} 42: 1)+\mathrm{H}$ & 1.6231 & 0.007 & 0.5924 & 1.3941 & 0.050 & -0.4197 \\
\hline 39 & $\mathrm{SM}(\mathrm{d} 22: 0 / 18: 1)$ & $\mathrm{SM}(\mathrm{d} 22: 0 / 18: 1)+\mathrm{HCOO}$ & 2.7354 & 0.001 & 0.6439 & 2.1077 & 0.001 & -0.3292 \\
\hline
\end{tabular}

VIP: variable important for the projection; FC: fold change; DG: diacylglycerol; MG: monoacylglycerol; CL: cardiolipin; FA: fatty acid; LPC: lysophosphatidylcholine; PC: phosphatidylcholine; LPE: lysophosphatidylethanolamine; PE: phosphatidylethanolamine; LPI: lysophosphatidylinositol; PS: phosphatidylserine; PG: phosphatidylglycerol; LPG: lysophosphatidylglycerol; SM: sphingomyelin. 


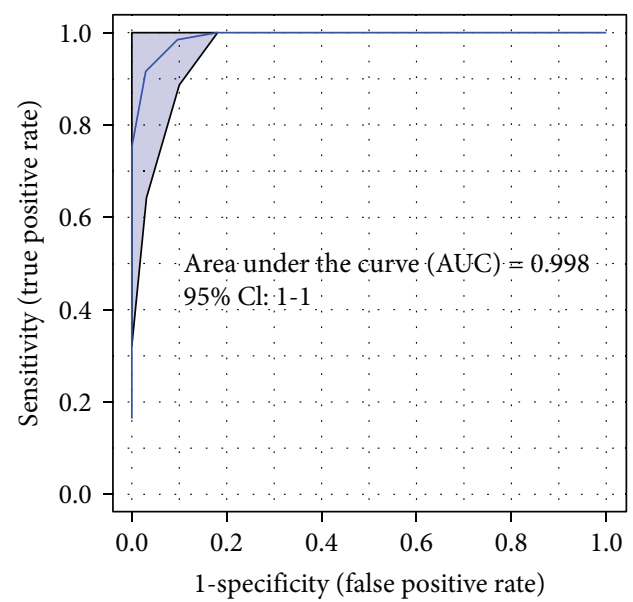

(a)

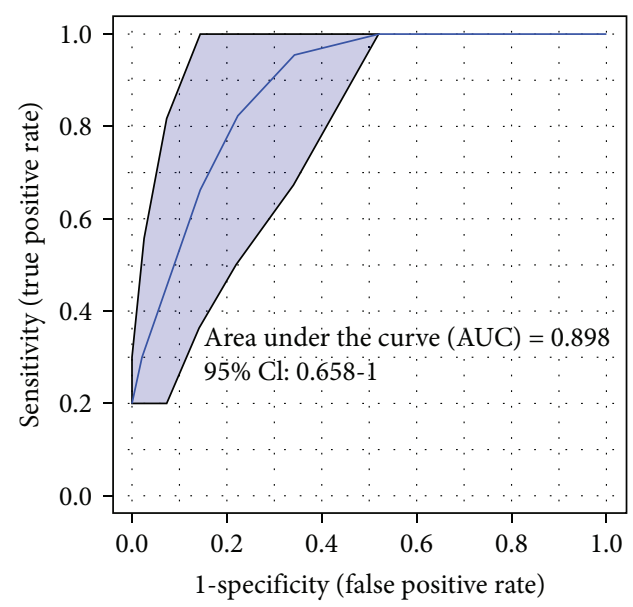

(c)

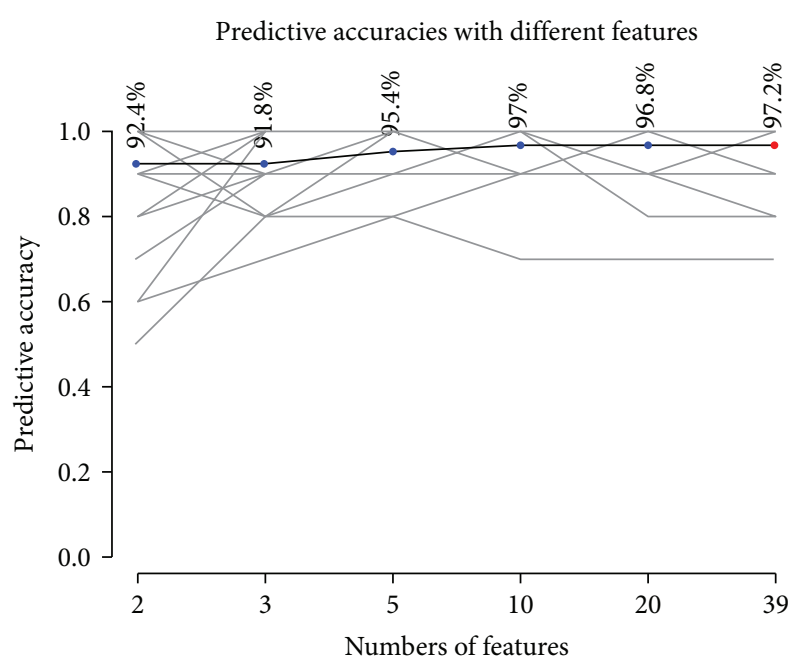

(b)

Predictive accuracies with different features

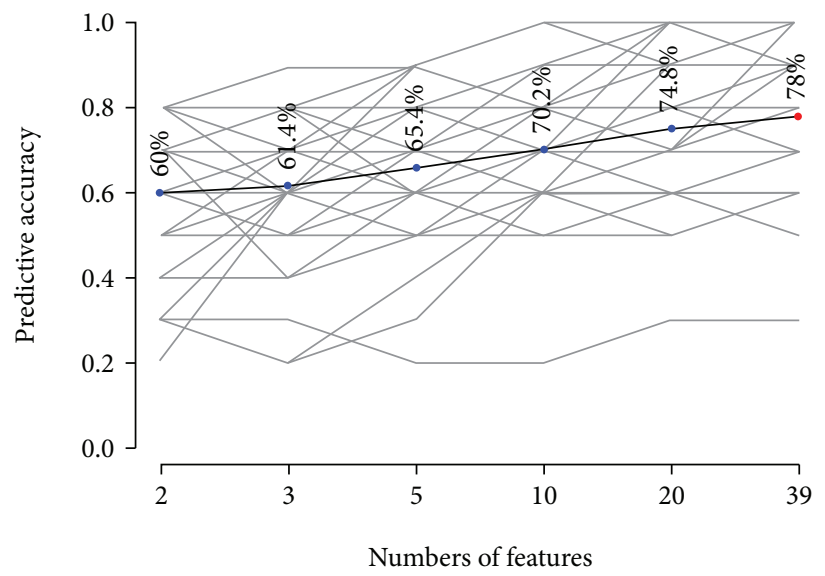

(d)

Figure 5

$\left(R^{2} X=0.500, R^{2} Y=0.994\right.$, and $Q^{2}=0.699$; Figure 4(a)) and positive $\quad\left(R^{2} X=0.564, \quad R^{2} Y=0.998, \quad\right.$ and $Q^{2}=0.649$; Figure 4(f)) ion modes, respectively. Compared to the control group, 140 negative ions contributed to clustering and discrimination which were extracted, of which 20 ions were downregulated and 120 ions were upregulated in the I/R group (Figures 4(b) and 4(e)). Similarly, 268 positive ions were selected as differential variables, among which, 21 ions were downregulated and 247 ions were upregulated in the I/R group (Figures 4(g) and 4(j)). Removing duplicate lipids, a total of 343 lipid species were identified from these differential ions indicating the lipidomic changes induced by $\mathrm{I} / \mathrm{R}$ injury in myocardium tissues.

To detect the modulation effect of astragaloside IV on lipid metabolism, OPLS-DA models were established between the drug-treated and I/R groups in the negative $\left(R^{2} X=0.376, R^{2} Y=0.965\right.$, and $Q^{2}=0.577$; Figure $\left.4(c)\right)$ and positive $\quad\left(R^{2} X=0.419, \quad R^{2} Y=0.916, \quad\right.$ and $Q^{2}=0.458$; Figure $4(\mathrm{~h})$ ) ion modes, respectively. As a result, 165 negative and 202 positive ions were extracted by the decided thresholds (Figures $4(\mathrm{~d})$ and $4(\mathrm{i})$ ). Compared to the I/R group, 95 negative and 126 positive ions were downregulated, while 70 negative and 76 positive ions were upregulated under the pretreatment of astragaloside IV (Figures 4(e) and 4(j)). In particular, Venn diagrams revealed that 22 abnormal negative and 24 positive ions induced by I/R injury could be regulated back to normal levels with the pretreatment of astragaloside IV. Since 5 lipids were commonly detected in both positive and negative ion modes and 2 lipids were in the formation of a quasimolecular ion and other adduct molecular ion in the positive mode, a total of 39 differential lipids were improved with a regression trend by astragaloside IV (Table 2).

We next evaluated the performance of 39 lipid features using ROC curve analysis. The results showed that with the increasing numbers of top lipids in use, diagnosis of samples from the I/R or the control group was obviously improved. When all the 39 lipids were employed, the AUC value was nearly 1.0 with $95 \%$ confidence interval from 1 to 1 (Figure 5(a)) and a predictive value of 97.2\% (Figure 5(b)). In the case of modeling between the astragaloside IVtreated group and the I/R group, the AUC value of 39 lipids 


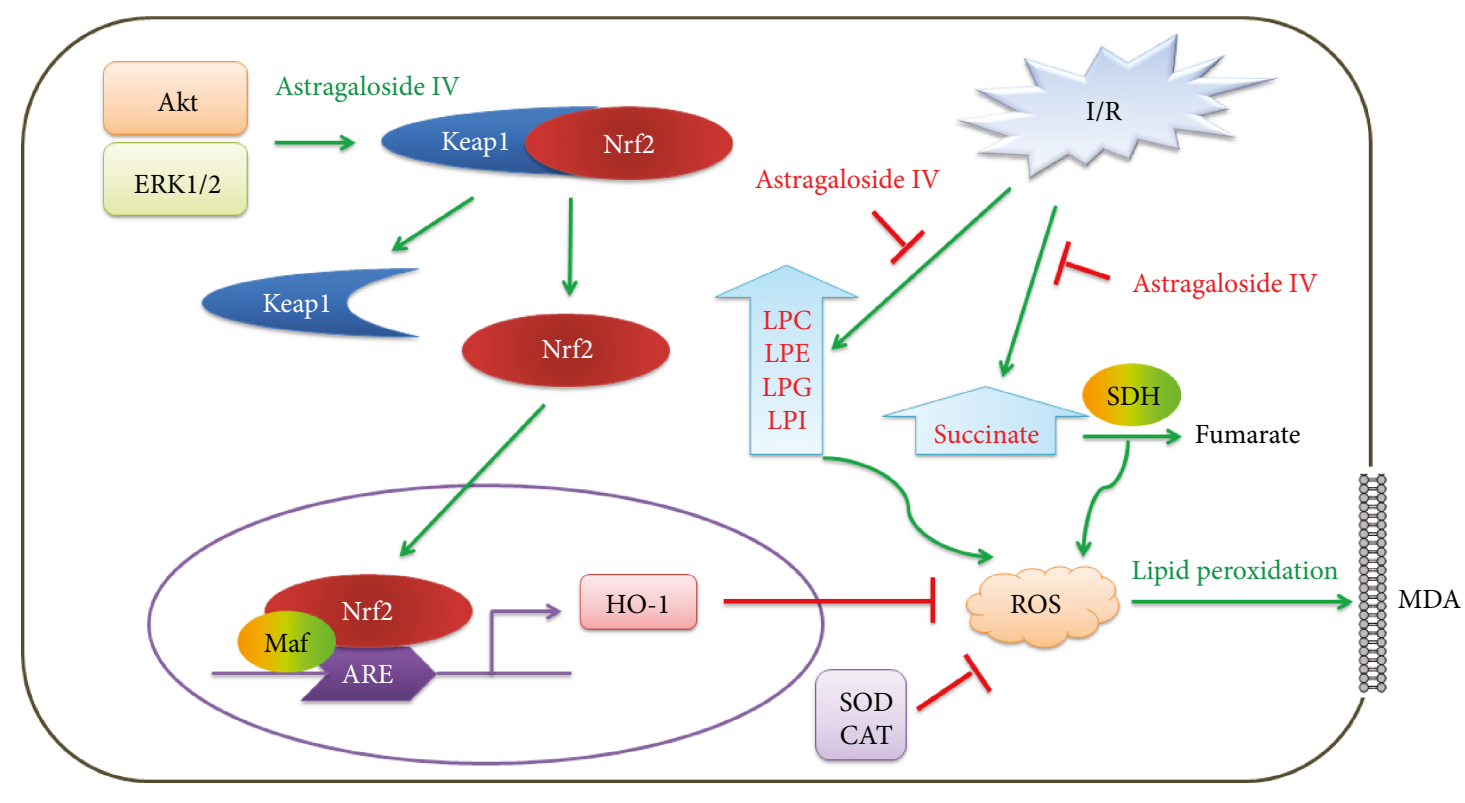

Figure 6

was 0.898 with $95 \%$ confidence interval from 0.658 to 1 (Figure 5(c)) and a predictive value of $78 \%$ (Figure $5(\mathrm{~d})$ ). These values indicated identified that these 39 lipids were important features to astragaloside and can prevent and improve myocardial I/R injury.

\section{Discussion}

In the present work, we studied the efficacy and antioxidative mechanism of astragaloside IV against myocardial I/R injury. Astragaloside IV significantly increased the coronary blood flow and heart rate (HR), left ventricular development pressure (LVDP), and maximal ascending/descending rate $\left( \pm \mathrm{d} p / \mathrm{d} t_{\max }\right)$ in the rats with $\mathrm{I} / \mathrm{R}$ injury. At the same time, astragaloside IV reduced the damage degree of myocardial cells by decreasing the myocardial enzyme levels of $\mathrm{CK}$ and $\mathrm{LDH}$ in the coronary effluent and plasma.

Nrf2 is a member of the leucine zipper (bZIP) protein family, and it is also an important transcription factor that regulates the expression of antioxidant proteins that protect against oxidative damage triggered by injury and inflammation [25]. Under quiescent conditions, Nrf2 is anchored in the cytoplasm through binding to Keap1, and its effect is repressed by Keap1 [26]. When oxidative stress activates the dissociation of Nrf2 and Keap1, Nrf2 translocates into the nucleus and binds to the antioxidant response element (ARE) in the upstream promoter region of many antioxidative genes and then initiates their transcription [27]. In myocardial I/R rat models, we found that I/R injury caused increasing expression levels of $\mathrm{Nrf} 2$ protein in both tissues and the nucleus, while astragaloside IV preconditioning facilitated Nrf2 translocating into the nucleus and then promoted Nrf2 expression in the nucleus. RT-qPCR results showed that I/R injury increased the mRNA level of Nrf2, whereas drug pretreatment decreased the level. Similarly, compared with the control group, the ratios of $\mathrm{p}$-Akt/Akt and $\mathrm{p}$ -
ERK1/2/ERK1/2 were significantly increased in the I/R group, and they were decreased after astragaloside IV preconditioning. Nrf2 is bound to Keap1 in the cytoplasm; once activated, it dissociates and enters the nucleus to bind to Maf for stimulating the expression of downstream protein $\mathrm{HO}-1$ [28]. As expected, Keap1 mRNA decreased in the I/R group compared with the control group, while it increased in the astragaloside IV pretreatment group $(p<0.01)$. Moreover, Maf and the downstream gene HO-1 in the I/R group were all significantly increased, while the SOD and CAT levels were lower than those in the control group. Notably, the expression of $\mathrm{Nrf} 2$ and $\mathrm{HO}-1$ proteins in the nucleus all increased, and it showed a more obvious trend after astragaloside IV preconditioning, suggesting astragaloside IV might activate the antioxidative pathway of Keap1/Nrf2.

SOD and CAT are important enzymes in organisms with the function of scavenging oxygen free radicals. In the I/R group, the SOD level significantly decreased whereas it increased after drug intervention. $\mathrm{SDH}$ is an enzyme involved in cell oxidation and mitochondrial oxidative phosphorylation in the tricarboxylic acid (TCA) cycle [29]. The I/R group showed a significant reduction of $\mathrm{SDH}$, which was improved in the drug-treated group, indicating that disturbance on TCA metabolism was prevented by astragaloside IV.

Succinate is an important metabolite of the TCA cycle. If a metabolic disorder occurs, it may affect the energy metabolism and related diseases of the body. It has been previously reported that succinate accumulates during ischemia, and the massive consumption of succinate during reperfusion can produce ROS [10]. Moreover, metabolomic analyses reveal that similar metabolic changes are observed in heart tissues of both ex vivo mouse Langendorff and in vivo ischemia and reperfusion experiments [10]. Since the ex vivo Langendorff heart is considered as a direct model for metabolomic analysis to observe ischemia and reperfusion 
injury, we thus employed ex vivo hearts to test the metabolic and lipidomic regulation effect of astragaloside IV. Using the ${ }^{1} \mathrm{H}$ NMR-based metabolomic method here, we found that succinate was significantly reduced after reperfusion, and metabolites in the relevant metabolic pathways for succinate production were also reduced, which suggested that succinate consumption was likely to produce a large amount of ROS in the damaged myocardium after subsequent reperfusion. Via preconditioning with astragaloside IV, succinate was obviously increased and other related metabolites also recovered obviously, indicating that astragaloside IV probably prevented the production of excessive ROS. Therefore, succinate metabolism can be used as a new therapeutic target for myocardial reperfusion injury [30].

Overproduction of active oxidizing free radicals during I/R injury may cause lipid peroxidation, which is broadly defined as a process of inserting hydroperoxy groups into lipids. Polyunsaturated fatty acids (PUFA) present in glycerolipids, glycerophospholipids, and cholesterols are often the targets of peroxidation, of which the peroxyl groups are derived from an oxygen molecule or from hydrogen peroxide [31]. Lipid peroxidation has been hypothesized to be a major mechanism of free radical damage. It may alter intrinsic membrane properties due to the physicochemical changes of oxidized lipids or indirectly contribute to other deleterious effects of ischemia/reperfusion through enhancing phospholipid susceptibility for degradation by phospholipases [32, 33] and increasing membrane calcium permeability [34]. MDA is the metabolic intermediate of lipid peroxidation and has been recognized as an indicator for lipid peroxidation. We found that MDA increased significantly in the model group, indicating that $\mathrm{I} / \mathrm{R}$ injury induced lipid peroxidation. Drug intervention decreased MDA obviously and revealed that astragaloside prevented lipid peroxidation by scavenging ROS.

Metabolomics has been an enabling technique for biomarker discovery and mechanism elucidation of coronary heart disease research [35]. Lipidomics, as a branch of metabolomics, has been used to investigate individual lipid species and their related metabolism pathway. A total of 39 lipids associated with $\mathrm{I} / \mathrm{R}$ injury were remarkably improved by astragaloside IV, including LPC $(15: 0,16: 0,16: 1 \mathrm{p}, 17: 0$, $18: 0,18: 1,18: 1 \mathrm{p}, 20: 1,20: 3,20: 4,22: 4$, and $22: 6$ ), PC $(16: 2 / 18: 2,17: 0 / 18: 2,36: 5,38: 4$, and $41: 4)$, LPE $(17: 0)$, PE (19:0e, 16:0/18:1, 18:0/20:5, and 39:5), LPG (16:0, $18: 1)$, PG $(17: 0 / 18: 1)$, LPI $(18: 1,18: 2$, and $20: 4)$, PS $(37: 3,40: 6)$, FA $(24: 5)$, CL $(18: 2 / 18: 2 / 20: 3 / 18: 2)$, MG (22:6), DG $(18: 1 / 22: 4,22: 6 / 22: 6)$, and SM (d39:1, d22:0/18:1, d42:1, and d22:1/20:1). Although these lipids increased or decreased in different degrees induced by $I / R$ injury, they were all regulated by astragaloside IV back to the normal levels of the control group. Among them, lysophospholipids (LPC, LPE, LPG, and LPI), as the products of plasmalogen reduction due to superoxide dismutase dysfunction, phospholipase activation, and oxidative stress [36-38], were found to be significantly downregulated in the drug-treated group, indicating astragaloside IV prevented oxidative stress damage and undue lipid peroxidation. The evidence was consistent with the results of biochemical fac- tors. However, the causes and mechanisms of some other lipid changes have not yet been understood and still need further investigation.

In summary, these results elucidate that astragaloside IV protects the myocardium from I/R injury through antioxidative stress, including preventing succinate accumulation to produce ROS, activating Nrf2 nuclear translocation to scavenge ROS, and avoiding lipid peroxidation caused by excessive ROS (Figure 6).

\section{Data Availability}

The data used to support the findings of this study are included within the article.

\section{Conflicts of Interest}

On behalf of all the authors, the corresponding author states that there is no conflict of interest.

\section{Acknowledgments}

This study was supported by the Tianjin Outstanding Youth Science Foundation (No. 17JCJQJC46200) and the National Natural Science Foundation of China (No. 81774050).

\section{References}

[1] G. A. Roth, M. D. Huffman, A. E. Moran et al., "Global and regional patterns in cardiovascular mortality from 1990 to 2013," Circulation, vol. 132, no. 17, pp. 1667-1678, 2015.

[2] B. Ibanez, S. James, S. Agewall et al., "2017 ESC Guidelines for the management of acute myocardial infarction in patients presenting with ST-segment elevation: The Task Force for the management of acute myocardial infarction in patients presenting with ST-segment elevation of the European Society of Cardiology (ESC)," European Heart Journal, vol. 39, no. 2, pp. 119-177, 2018.

[3] D. M. Yellon and D. J. Hausenloy, "Myocardial reperfusion injury," The New England Journal of Medicine, vol. 357, no. 11, pp. 1121-1135, 2007.

[4] M. Ovize, H. Thibault, and K. Przyklenk, "Myocardial conditioning: opportunities for clinical translation," Circulation Research, vol. 113, no. 4, pp. 439-450, 2013.

[5] D. J. Lefer and D. N. Granger, "Oxidative stress and cardiac disease," The American Journal of Medicine, vol. 109, no. 4, pp. 315-323, 2000.

[6] H. K. Eltzschig and T. Eckle, "Ischemia and reperfusion-from mechanism to translation," Nature Medicine, vol. 17, no. 11, pp. 1391-1401, 2011.

[7] L. Timmers, G. Pasterkamp, V. C. de Hoog, F. Arslan, Y. Appelman, and D. P. V. de Kleijn, "The innate immune response in reperfused myocardium," Cardiovascular Research, vol. 94, no. 2, pp. 276-283, 2012.

[8] D. Roul and F. A. Recchia, "Metabolic alterations induce oxidative stress in diabetic and failing hearts: different pathways, same outcome," Antioxidants \& Redox Signaling, vol. 22, no. 17, pp. 1502-1514, 2015.

[9] M. Board, C. Lopez, C. van den Bos, R. Callaghan, K. Clarke, and C. Carr, "Acetoacetate is a more efficient energy-yielding substrate for human mesenchymal stem cells than glucose and 
generates fewer reactive oxygen species," The International Journal of Biochemistry \& Cell Biology, vol. 88, pp. 75-83, 2017.

[10] E. T. Chouchani, V. R. Pell, E. Gaude et al., "Ischaemic accumulation of succinate controls reperfusion injury through mitochondrial ROS," Nature, vol. 515, no. 7527, pp. 431-435, 2014.

[11] R. J. Mailloux, A. Young, J. Chalker et al., "Choline and dimethylglycine produce superoxide/hydrogen peroxide from the electron transport chain in liver mitochondria," FEBS Letters, vol. 590, no. 23, pp. 4318-4328, 2016.

[12] S. A. Coolen, C. A. Daykin, J. P. M. van Duynhoven et al., "Measurement of ischaemia-reperfusion in patients with intermittent claudication using NMR-based metabonomics," NMR in Biomedicine, vol. 21, no. 7, pp. 686-695, 2008.

[13] X. Cheng, J. Gu, M. Zhang et al., "Astragaloside IV inhibits migration and invasion in human lung cancer A549 cells via regulating PKC- $\alpha$-ERK1/2-NF- $\kappa \mathrm{B}$ pathway," International Immunopharmacology, vol. 23, no. 1, pp. 304-313, 2014.

[14] W. J. Zhang and B. Frei, "Astragaloside IV inhibits NF- $\kappa$ B activation and inflammatory gene expression in LPS-treated mice," Mediators of Inflammation, vol. 2015, Article ID 274314, 11 pages, 2015.

[15] D. Gui, J. Huang, W. Liu, Y. Guo, W. Xiao, and N. Wang, "Astragaloside IV prevents acute kidney injury in two rodent models by inhibiting oxidative stress and apoptosis pathways," Apoptosis, vol. 18, no. 4, pp. 409-422, 2013.

[16] L. Lv, S. Y. Wu, G. F. Wang et al., "Effect of astragaloside IV on hepatic glucose-regulating enzymes in diabetic mice induced by a high-fat diet and streptozotocin," Phytotherapy Research, vol. 24, no. 2, pp. 219-224, 2010.

[17] H. L. Wang, Q. H. Zhou, M. B. Xu, X. L. Zhou, and G. Q. Zheng, "Astragaloside IV for experimental focal cerebral ischemia: preclinical evidence and possible mechanisms," Oxidative Medicine and Cellular Longevity, vol. 2017, Article ID 8424326, 13 pages, 2017.

[18] S. Ren, H. Zhang, Y. Mu, M. Sun, and P. Liu, "Pharmacological effects of astragaloside IV: a literature review," Journal of Traditional Chinese Medicine, vol. 33, no. 3, pp. 413-416, 2013.

[19] X. H. Han, P. Liu, Y. Y. Zhang, N. Zhang, F. R. Chen, and J. F. Cai, "Astragaloside IV regulates expression of ATP-sensitive potassium channel subunits after ischemia-reperfusion in rat ventricular cardiomyocytes," Journal of Traditional Chinese Medicine, vol. 31, no. 4, pp. 321-326, 2011.

[20] L. Tu, C. S. Pan, X. H. Wei et al., "Astragaloside IV protects heart from ischemia and reperfusion injury via energy regulation mechanisms," Microcirculation, vol. 20, no. 8, pp. 736747, 2013.

[21] S. G. Wang, Y. Xu, J. D. Chen, C. H. Yang, and X. H. Chen, "Astragaloside IV stimulates angiogenesis and increases nitric oxide accumulation via JAK2/STAT3 and ERK1/2 pathway," Molecules, vol. 18, no. 10, pp. 12809-12819, 2013.

[22] J. Si, N. Wang, H. Wang et al., "HIF- $1 \alpha$ signaling activation by post-ischemia treatment with astragaloside IV attenuates myocardial ischemia-reperfusion injury," PLoS One, vol. 9, no. 9, article e107832, 2014.

[23] Y. He, J. Xi, H. Zheng, Y. Zhang, Y. Jin, and Z. Xu, “Astragaloside IV inhibits oxidative stress-induced mitochondrial permeability transition pore opening by inactivating GSK-3 $\beta$ via nitric oxide in $\mathrm{H} 9 \mathrm{c} 2$ cardiac cells," Oxidative Medicine and Cellular Longevity, vol. 2012, Article ID 935738, 9 pages, 2012.

[24] E. Cequier-Sánchez, C. Rodríguez, Á. G. Ravelo, and R. Zárate, "Dichloromethane as a solvent for lipid extraction and assess- ment of lipid classes and fatty acids from samples of different natures," Journal of Agricultural and Food Chemistry, vol. 56, no. 12, pp. 4297-4303, 2008.

[25] R. Gold, L. Kappos, D. L. Arnold et al., "Placebo-controlled phase 3 study of oral BG-12 for relapsing multiple sclerosis," The New England Journal of Medicine, vol. 367, no. 12, pp. 1098-1107, 2012.

[26] P. Moi, K. Chan, I. Asunis, A. Cao, and Y. W. Kan, "Isolation of NF-E2-related factor 2 (Nrf2), a NF-E2-like basic leucine zipper transcriptional activator that binds to the tandem NFE2/AP1 repeat of the beta-globin locus control region," Proceedings of the National Academy of Sciences of the United States of America, vol. 91, no. 21, pp. 9926-9930, 1994.

[27] T. Yamamoto, T. Suzuki, A. Kobayashi et al., "Physiological significance of reactive cysteine residues of Keap1 in determining Nrf2 activity," Molecular and Cellular Biology, vol. 28, no. 8, pp. 2758-2770, 2008.

[28] K. Itoh, T. Chiba, S. Takahashi et al., “An Nrf2/small Maf heterodimer mediates the induction of phase II detoxifying enzyme genes through antioxidant response elements," Biochemical and Biophysical Research Communications, vol. 236, no. 2, pp. 313-322, 1997.

[29] K. S. Oyedotun and B. D. Lemire, "The quaternary structure of the Saccharomyces cerevisiae succinate dehydrogenase. Homology modeling, cofactor docking, and molecular dynamics simulation studies," The Journal of Biological Chemistry, vol. 279, no. 10, pp. 9424-9431, 2004.

[30] V. R. Pell, E. T. Chouchani, C. Frezza, M. P. Murphy, and T. Krieg, "Succinate metabolism: a new therapeutic target for myocardial reperfusion injury," Cardiovascular Research, vol. 111, no. 2, pp. 134-141, 2016.

[31] T. S. Anthonymuthu, E. M. Kenny, and H. Bayır, "Therapies targeting lipid peroxidation in traumatic brain injury," Brain Research, vol. 1640, Part A, pp. 57-76, 2016.

[32] A. Sevanian, R. A. Stein, and J. F. Mead, "Metabolism of epoxidized phosphatidylcholine by phospholipase A2 and epoxide hydrolase," Lipids, vol. 16, no. 11, pp. 781-789, 1981.

[33] W. B. Weglicki, B. F. Dickens, and I. T. Mak, "Enhanced lysosomal phospholipid degradation and lysophospholipid production due to free radicals," Biochemical and Biophysical Research Communications, vol. 124, no. 1, pp. 229-235, 1984.

[34] D. Bagchi, G. J. Wetscher, M. Bagchi et al., "Interrelationship between cellular calcium homeostasis and free radical generation in myocardial reperfusion injury," Chemico-Biological Interactions, vol. 104, no. 2-3, pp. 65-85, 1997.

[35] Y. Fan, Y. Li, Y. Chen et al., "Comprehensive metabolomic characterization of coronary artery diseases," Journal of the American College of Cardiology, vol. 68, no. 12, pp. 12811293, 2016.

[36] N. Nagan and R. A. Zoeller, "Plasmalogens: biosynthesis and functions," Progress in Lipid Research, vol. 40, no. 3, pp. 199229, 2001.

[37] R. W. Gross, "High plasmalogen and arachidonic acid content of canine myocardial sarcolemma: a fast atom bombardment mass spectroscopic and gas chromatography-mass spectroscopic characterization," Biochemistry, vol. 23, no. 1, pp. 158-165, 2002.

[38] J. Lu, B. Chen, T. Chen et al., "Comprehensive metabolomics identified lipid peroxidation as a prominent feature in human plasma of patients with coronary heart diseases," Redox Biology, vol. 12, pp. 899-907, 2017. 


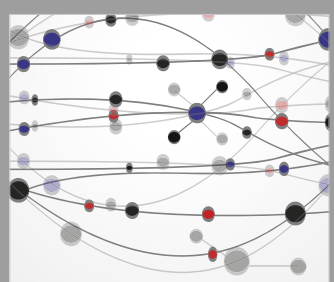

The Scientific World Journal
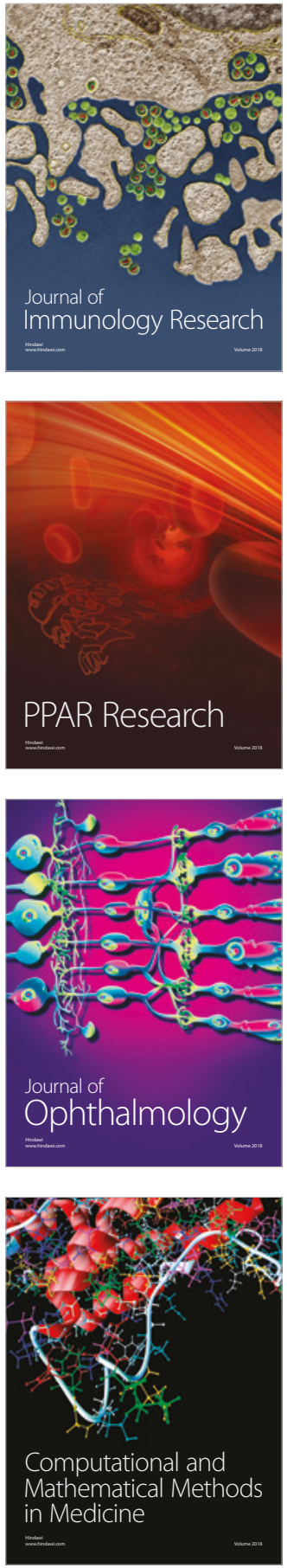

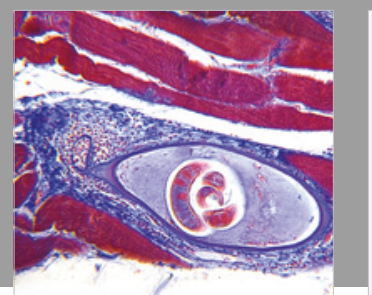

Gastroenterology Research and Practice

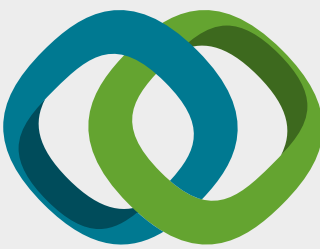

\section{Hindawi}

Submit your manuscripts at

www.hindawi.com
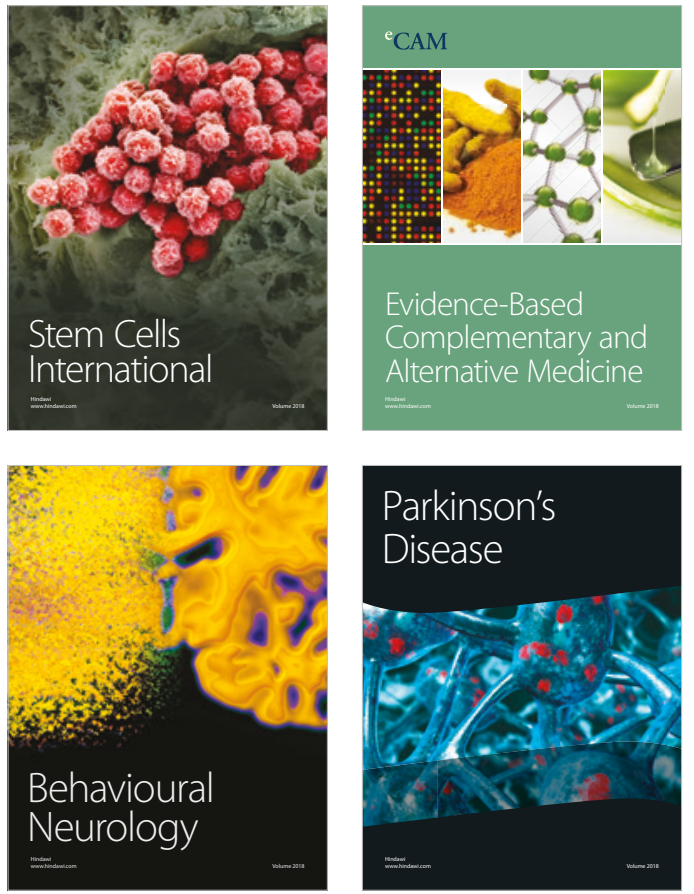

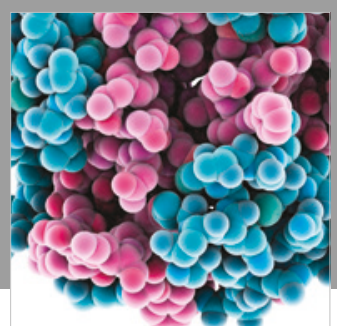

ournal of

Diabetes Research

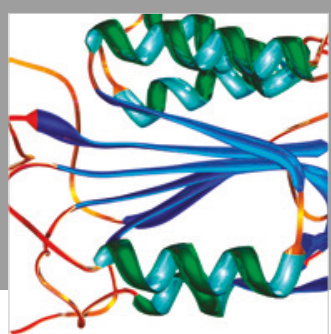

Disease Markers
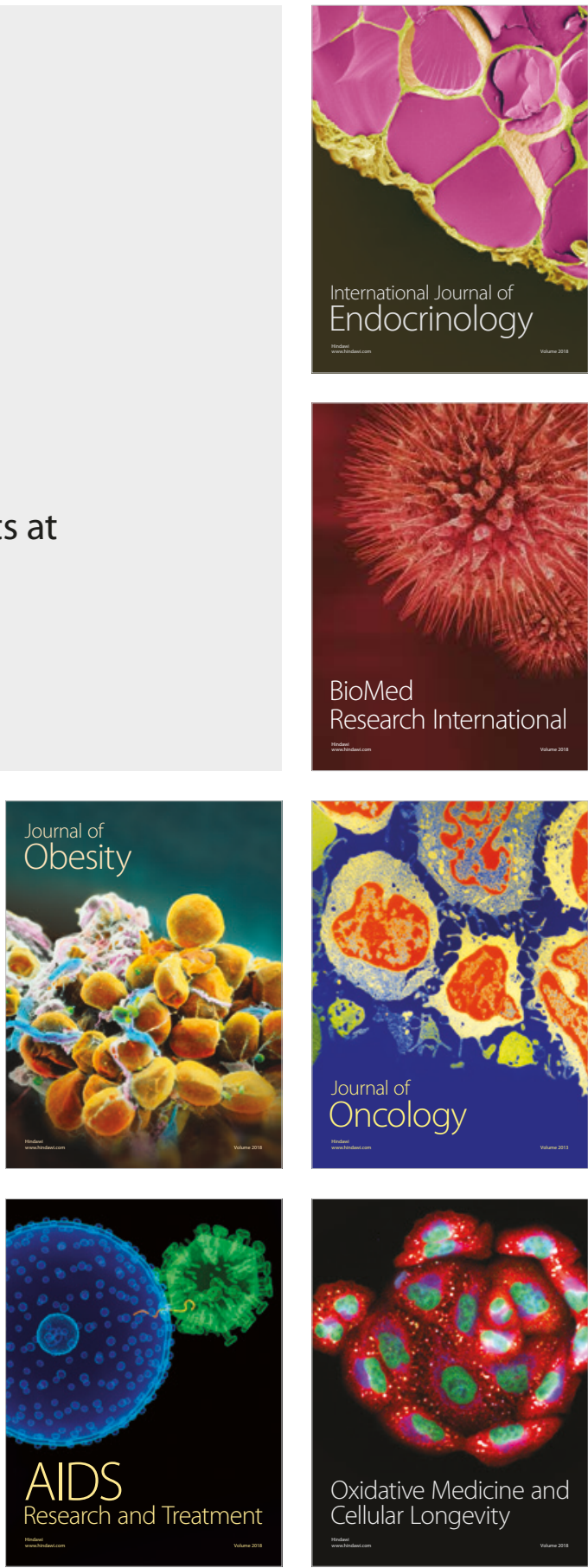